TRANSACTIONS OF THE

AMERICAN MATHEMATICAL SOCIETY

Volume 349, Number 1, January 1997, Pages 311-330

S 0002-9947(97)01755-8

\title{
AUTOMORPHISM GROUPS AND INVARIANT SUBSPACE LATTICES
}

\author{
PAUL S. MUHLY AND BARUCH SOLEL
}

\begin{abstract}
Let $(B, \mathbf{R}, \alpha)$ be a $C^{*}$ - dynamical system and let $A=B^{\alpha}([0, \infty))$ be the analytic subalgebra of $B$. We extend the work of Loebl and the first author that relates the invariant subspace structure of $\pi(A)$, for a $C^{*}$ representation $\pi$ on a Hilbert space $\mathcal{H}_{\pi}$, to the possibility of implementing $\alpha$ on $\mathcal{H}_{\pi}$. We show that if $\pi$ is irreducible and if lat $\pi(A)$ is trivial, then $\pi(A)$ is ultraweakly dense in $\mathcal{L}\left(\mathcal{H}_{\pi}\right)$. We show, too, that if $A$ satisfies what we call the strong Dirichlet condition, then the ultraweak closure of $\pi(A)$ is a nest algebra for each irreducible representation $\pi$. Our methods give a new proof of a "density" theorem of Kaftal, Larson, and Weiss and they sharpen earlier results of ours on the representation theory of certain subalgebras of groupoid $C^{*}$-algebras.
\end{abstract}

\section{INTRODUCTION}

Throughout this note, $(B, \mathbf{R}, \alpha)$ will be a fixed $C^{*}$-dynamical system determined by a separable ${ }^{1} C^{*}$-algebra $B$ and a strongly continuous representation $\alpha=\left\{\alpha_{t}\right\}$ of $\mathbf{R}$ on $B$ as a one-parameter group of $*$-automorphisms. We let $A$ denote the subalgebra consisting of the analytic elements in $B$ with respect to $\alpha$. Thus $A$ consists of all those $a \in B$ such that $t \rightarrow \alpha_{t}(a)$ is an $H^{\infty}(\mathbf{R}), B$-valued function on R. Alternatively, $A$ is the spectral subspace $B^{\alpha}[0, \infty)$. (See [3], [17] and [23].) We call $A$ the analytic subalgebra of $B$ determined by $\alpha$. We are interested in the following general problem: Given a $C^{*}$-representation $\pi$ of $B$, how are the properties of the $C^{*}$-dynamical system $(B, \mathbf{R}, \alpha)$ reflected in the invariant subspace structure of $\pi(A)$ ? One of the first consequences of our analysis is

Theorem 1.1. If $\pi$ is an irreducible representation of $B$ on a Hilbert space $\mathcal{H}_{\pi}$ and if $\pi(A)$ has no nontrivial invariant subspaces, then $\pi(A)$ is ultraweakly dense in $\mathcal{L}\left(\mathcal{H}_{\pi}\right)$.

Received by the editors July 11, 1994 and, in revised form, October 8, 1995.

1991 Mathematics Subject Classification. Primary 46K50, 47D25, 47D99, 46L40; Secondary 46L50, 46L55, 46L99.

Supported in part by grants from the U. S. National Science Foundation and the U. S. - Israel Binational Science Foundation.

Supported in part by the U. S. - Israel Binational Science Foundation and the Fund for the Promotion of Research at the Technion.

${ }^{1}$ While separability is not critical for everything we do, it is important at a number of key points. Therefore we make this blanket assumption at the outset. Most of our representations will be on separable Hilbert spaces, too. However, we do have to make contact with the "nonseparable" through the intervention of universal representations. We shall be careful about this and signal that a given representation acts on a separable space either by calling the space separable or by saying that the representation is separable. 
An algebra $\mathcal{A}$ of operators on a Hilbert space $\mathcal{H}$ is called transitive if it has no nontrivial invariant subspaces. The transitive algebra problem is to determine if there are any proper, weakly closed transitive operator algebras. Theorem 1.1 shows that none can be constructed from analytic operator algebras. More important, perhaps, is the new perspective Theorem 1.1 places on the initial construction of an irreducible triangular operator algebra due to Kadison and Singer [14] and its refinement due to Arveson [2]. These algebras can be described in terms of $C^{*}$ dynamical systems and Theorem 1.1 provides a new proof that they are ultraweakly dense. In earlier work, the ultraweak density is derived as a consequence of the fact that the algebra in question contains a masa; our proof uses, instead, the harmonic analysis of $C^{*}$-dynamical systems.

Our analysis rests fundamentally on [17], where it is shown how the invariant subspace structure of the analytic subalgebra of a $W^{*}$-dynamical system is tied to the covariant representations of the system. The improvements to [17] that this note provides yield refined information about the full lattice of invariant subspaces of $\pi(A)$. This is illustrated by Theorem 1.2 .

The key assumption that we need is that $A$ is strongly Dirichlet in the following sense. We write $B^{\alpha}(-\infty, 0)$ for the norm closure of the space of elements $b$ in $B$ such that the spectrum of $b, s p_{\alpha}(b)$, is compact and contained in the interval $(-\infty, 0)$. Likewise, we define $B^{\alpha}(0, \infty)$. The space $B^{\alpha}(0)$ consists of those elements $b$ in $B$ such that $s p_{\alpha}(b) \subseteq\{0\}$. Alternatively, $B^{\alpha}(0)$ is the fixed point algebra of $\alpha$. Evidently, $B^{\alpha}(0)$ and $B^{\alpha}(0, \infty)$ are both contained in $A$ and $B^{\alpha}(0, \infty)$ is a bimodule over $B^{\alpha}(0)$, but $A$ may be larger than their span. Also, $B^{\alpha}(-\infty, 0)^{*}=B^{\alpha}(0, \infty)$. We shall say that $A$ is a strongly Dirichlet subalgebra of $B$ in case $B$ is the closure in norm of the space $B^{\alpha}(-\infty, 0)+B^{\alpha}(0)+B^{\alpha}(0, \infty)$. As we shall show in $\S 3$ below, the assumption that $A$ is a strongly Dirichlet subalgebra of $B$ is equivalent to assuming that $\alpha$ is uniformly averaging in the sense that there is a faithful, $\alpha$-invariant, conditional expectation from $B$ onto $B^{\alpha}(0)$ such that

$$
\lim _{T \rightarrow \infty} \frac{1}{2 T} \int_{-T}^{T} \alpha_{t}(b) d t=\Phi(b)
$$

in the norm topology for each $b \in B$. It is unknown how much stronger the assumption is that $A$ is a strongly Dirichlet subalgebra of $B$ than the assumption that $A$ is a Dirichlet subalgebra of $B$, meaning that $A+A^{*}$ is norm dense in $B$.

Theorem 1.2. If $A$ is a strongly Dirichlet subalgebra of $B$ and if $\pi$ is an irreducible representation of $B$ on a Hilbert space $\mathcal{H}_{\pi}$, then there is a nest $\mathcal{N}$ of subspaces of $\mathcal{H}_{\pi}$ such that the ultraweak closure of $\pi(A)$ is the nest algebra $\operatorname{alg}(\mathcal{N})$, where $\operatorname{alg}(\mathcal{N})$ is the set of all operators on $\mathcal{H}_{\pi}$ that leave each subspace in $\mathcal{N}$ invariant.

In a sense Theorem 1.2 contains Theorem 1.1. Theorem 1.1 says that (without any hypotheses on $A$ ) if $\pi(A)$ has no invariant subspaces, then $\pi(A)$ is ultraweakly dense in $\operatorname{alg}(\mathcal{N})$ where $\mathcal{N}$ is the trivial nest, $\{0, I\}$. Also, it should be noted that the techniques of [17] always produce a whole nest $\mathcal{N}$ of invariant subspaces for $\pi(A)$ whenever there is a nontrivial invariant subspace. Consequently, $\pi(A) \subseteq \operatorname{alg}(\mathcal{N})$. The point of the hypothesis that $A$ is a strongly Dirichlet subalgebra of $B$ is to guarantee that the ultraweak closure of $\pi(A)$ equals $\operatorname{alg}(\mathcal{N})$.

Theorems 1.1 and 1.2 are proved in the next section. Section 3 has two objectives. First, we investigate the relation between the strong Dirichlet property and the uniform averaging condition. This was studied in [20] using some results of 
Jørgensen [13]. However, one of the results in [13] that was used is incorrect. In $\S 3$ we fix the error in [13] and the proof in [20] where it was used. As our second objective, we use the analysis that went to repair [13] coupled with the techniques developed in $\S 2$ to provide a new proof of Theorem 10 in [15], which shows that a nest subalgebra of a semifinite von Neumann algebra is "local". More precisely, if $\mathcal{B}$ is such a von Neumann algebra, with ideal of relatively compact operators $\mathcal{K}$, and if $\mathcal{A}=\mathcal{B} \cap \operatorname{alg}(\mathcal{N})$, where $\mathcal{N}$ is a nest whose projections lie in $\mathcal{B}$, then $\mathcal{A} \cap \mathcal{K}$ is ultraweakly dense in $\mathcal{A}$. In Section 4 we use Theorems 1.1 and 1.2 and the analysis that enters into their proofs to complement the results of [21]. In the setting of [21], $B=C^{*}(G ; E)$, where $E$ is a $\mathbf{T}$-groupoid over an $r$-discrete, principal, amenable groupoid $G$. By virtue of Kumjian's representation theorem [16], such an algebra $B$ may be described in a coordinate free way as a nuclear $C^{*}$-algebra containing a diagonal. The automorphism group $\alpha$ is determined by a cocycle $c$ on $G$ and so, therefore, $A$ is determined by $c$. The invariant subspace lattice of $\pi(A)$, lat $(\pi(A))$, turns out to be intimately tied to the asymptotic behavior of $c$. Our analysis of this relation answers some questions raised in [21]; it gives new proofs and extensions of some results of Orr and Peters [22]; and it provides the new perspective on the results of Kadison and Singer [14] and Arveson [1] alluded to above.

Acknowledgement. We wish to thank László Zsidó for calling our attention to his paper [27], which helped to simplify some arguments in a first draft of this paper as well as to provide an alternate approach to some of the discussion presented here.

\section{Representations AND InVARIANT SUBSpaCES}

The terminology of the theory of $C^{*}$-dynamical systems is not universally agreed upon. Since certain fine points in the theory are important for our analysis, we begin with some definitions and relate them to the terminology in the two basic references that we follow, [10] and [23].

Following [23], we call a pair $(\pi, U)$ a covariant representation of the dynamical system $(B, \mathbf{R}, \alpha)$ if $\pi$ is a representation of $B$ on a Hilbert space $\mathcal{H}_{\pi}$ and $U=$ $\left\{U_{t}\right\}_{t \in \mathbf{R}}$ is a unitary representation of $\mathbf{R}$ on $\mathcal{H}_{\pi}$ such that the equation

$$
\pi\left(\alpha_{t}(a)\right)=U_{t} \pi(a) U_{t}^{*}
$$

holds for all $t \in \mathbf{R}$ and $a \in B$. A covariant representation $(\pi, U)$ of $(B, \mathbf{R}, \alpha)$ may be integrated to yield a $C^{*}$-representation $\pi \times U$ of the $C^{*}$-crossed product, $B \times{ }_{\alpha} \mathbf{R}$. And conversely, each $C^{*}$-representation of $B \times{ }_{\alpha} \mathbf{R}$ may be disintegrated in terms of a covariant representation of $(B, \mathbf{R}, \alpha)$. See $\S 7.6$ of [23].

A $C^{*}$-representation $\pi$ of $B$ on a Hilbert space $\mathcal{H}_{\pi}$ will be called protocovariant in case there is a unitary representation of $\mathbf{R}, U=\left\{U_{t}\right\}_{t \in \mathbf{R}}$ on $\mathcal{H}_{\pi}$ such that $(\pi, U)$ is a covariant representation of $(B, \mathbf{R}, \alpha)$. Guichardet [10] calls such a $\pi$ covariant, but Pedersen does not assign any special term to it. Of course given a (separable) protocovariant representation $\pi$ of $B$, the unitary representations $U=\left\{U_{t}\right\}_{t \in \mathbf{R}}$ that one may take to build covariant representations of $(B, \mathbf{R}, \alpha)$ are "affinely related" by 1-cocycles for $\mathbf{R}$ with values in the unitary group of $\pi(B)^{\prime}$. The distinction we wish to emphasize is that covariant representations of $(B, \mathbf{R}, \alpha)$ are tied to the representations of $B \times{ }_{\alpha} \mathbf{R}$. As we have indicated, they really are the representations of $B \times{ }_{\alpha} \mathbf{R}$. Protocovariant representations, on the other hand, are representations of $B$. 
We call a representation of $B$ quasi-covariant if it is quasi-equivalent to a protocovariant representation of $B$. (Recall from $\S 5.3$ of [9] that two representations $\pi_{1}$ and $\pi_{2}$ of $B$ are quasi-equivalent in case there is an isomorphism $\Phi$ from $\pi_{1}(B)^{\prime \prime}$ onto $\pi_{2}(B)^{\prime \prime}$ such that $\pi_{2}(b)=\Phi\left(\pi_{1}(b)\right)$ for all $b \in B$.) Guichardet [10] calls a representation $\pi$ of $B$ quasi-invariant in case $\pi$ and $\pi \circ \alpha_{t}$ are quasi-equivalent for all $t$. He shows in Proposition X.3 of [10] that a separable quasi-invariant representation is quasi-covariant in our sense. On the other hand, Pedersen calls a separable quasi-covariant representation R-invariant. (See Theorem 7.4.10 of [23]. Note, too, that he uses the term 'equivalent' for 'quasi-equivalent'.)

The difference between the notions of protocovariance and quasi-covariance is one, really, of multiplicity, as the following proposition shows.

Proposition 2.1. Let $\pi$ be $a C^{*}$-representation of $B$ on a separable Hilbert space $\mathcal{H}$. Then the following assertions are equivalent.

1) $\pi$ is quasi-covariant.

2) There is an ultraweakly continuous representation of $\mathbf{R}$ on $\pi(B)^{\prime \prime},\left\{\beta_{t}\right\}_{t \in \mathbf{R}}$, such that $\beta_{t} \circ \pi=\pi \circ \alpha_{t}$, for all $t$.

3) An infinite multiple of $\pi, \infty \cdot \pi$, is protocovariant.

Proof. 1) $\Rightarrow 2$ ). If $\pi$ is quasi-covariant, then there are a covariant representation $(\rho, V)$ of $(B, \mathbf{R}, \alpha)$ and an isomorphism $\Psi: \pi(B)^{\prime \prime} \rightarrow \rho(B)^{\prime \prime}$ such that $\Psi \circ \pi=\rho$. If $\beta_{t}$ is defined to be $\Psi^{-1}\left(A d V_{t}\right) \Psi$, then $\left\{\beta_{t}\right\}_{t \in \mathbf{R}}$ is an ultraweakly continuous representation of $\mathbf{R}$ on $\pi(B)^{\prime \prime}$ such that $\beta_{t} \circ \pi=\pi \circ \alpha_{t}$, for all $t \in \mathbf{R}$.

$2) \Rightarrow 3)$. Suppose that there is an ultraweakly continuous representation of $\mathbf{R}$ on $\pi(B)^{\prime \prime},\left\{\beta_{t}\right\}_{t \in \mathbf{R}}$, such that $\beta_{t} \circ \pi=\pi \circ \alpha_{t}$ for all $t$. Then since ampliations are von Neumann algebra isomorphisms, we may replace $\pi$ by $\infty \cdot \pi$ acting on $\mathcal{H}_{\pi} \otimes \ell^{2}(\mathbf{Z})$ and assume that $\left\{\beta_{t}\right\}_{t \in \mathbf{R}}$ acts on $(\infty \cdot \pi)(B)^{\prime \prime}$ and satisfies the equation $\beta_{t} \circ(\infty \cdot \pi)=(\infty \cdot \pi) \circ \alpha_{t}$, for all $t$. The von Neumann algebra $(\infty \cdot \pi)(B)^{\prime \prime}$ acts on a separable space and has a properly infinite commutant. In Corollary 10 of [12], Halpern shows that if $(\infty \cdot \pi)(B)^{\prime \prime}$ were semifinite, then $\left\{\beta_{t}\right\}_{t \in \mathbf{R}}$ would be spatially implemented. However, as we show, his argument is quite general, thanks to work of Haagerup [11] and others (see [6], §2.5.4). Indeed, write $\mathcal{B}$ for $(\infty \cdot \pi)(B)^{\prime \prime}$. There is a von Neumann algebra isomorphism $\rho: \mathcal{B} \rightarrow \widetilde{\mathcal{B}}$, where $\widetilde{\mathcal{B}}$ acts on a separable space $\mathcal{H}_{\rho}$ such that $\widetilde{\mathcal{B}}$ has a separating and cyclic vector; i.e., $\widetilde{\mathcal{B}}$ is in standard form. By [11, Theorem 3.2 and Corollary 3.6], there is a unitary representation of $\mathbf{R},\left\{V_{t}\right\}_{t \in \mathbf{R}}$, on $\mathcal{H}_{\rho}$ such that $\rho \circ \beta_{t}=\left(A d V_{t}\right) \circ \rho$ for all $t$. Form $\rho \otimes I$, mapping $\mathcal{B}$ to $\widetilde{\mathcal{B}} \otimes \mathbf{C} I$ acting on $\mathcal{H}_{\rho} \otimes \ell^{2}(\mathbf{Z})$, where $I$ is the identity operator on $\ell^{2}(\mathbf{Z})$. The commutant $(\widetilde{\mathcal{B}} \otimes \mathbf{C} I)^{\prime}=\widetilde{\mathcal{B}}^{\prime} \otimes \mathcal{L}\left(\ell^{2}(\mathbf{Z})\right)$ is also properly infinite (and acts on a separable space) and so by Corollary 8 in $\S 8$ of Chapter III of [8], $\rho \otimes I$ is spatial. However, we have $(\rho \otimes I) \circ \beta_{t}=\left(A d\left(V_{t} \otimes I\right)\right) \circ(\rho \otimes I)$ and this shows that $\left\{\beta_{t}\right\}_{t \in \mathbf{R}}$ is spatially implemented on $\mathcal{H}_{\pi} \otimes \ell^{2}(\mathbf{Z})$. Thus $\infty \cdot \pi$ is protocovariant.

$3) \Rightarrow 1$ ). This is clear, since $\infty \cdot \pi$ is quasi-equivalent to $\pi, \infty \cdot \pi$ is quasi-covariant, and quasi-covariance is preserved under quasi-equivalence.

Our next objective is to identify the largest central summand of the universal enveloping von Neumann of $B$ that "carries" all the quasi-covariant representations of $B$. To this end, recall that the covariant representations of $(B, \mathbf{R}, \alpha)$ are in one-toone correspondence with the $C^{*}$-representations of the crossed product $C^{*}$-algebra, $B \times{ }_{\alpha} \mathbf{R}$. (See $\S 7.6$ of [23].) Let $\tilde{\Phi}$ be the universal representation of $B \times{ }_{\alpha} \mathbf{R}$ and let $\Phi$ be the universal representation of $B$. (Thus $\Phi(B)^{\prime \prime}$ is the universal enveloping 
von Neumann algebra of $B$.) By virtue of the correspondence between covariant representations of $(B, \mathbf{R}, \alpha)$ and representations of $B \times{ }_{\alpha} \mathbf{R}$, we may write $\tilde{\Phi}=\pi_{0} \times U$ for a certain covariant representation $\left(\pi_{0}, U\right)$ of $(B, \mathbf{R}, \alpha)$. Let $c_{0}=c\left(\pi_{0}\right)$ be the central support of $\pi_{0}$ in $\Phi(B)^{\prime \prime}$ (also called the central cover of $\pi_{0}$ ) and write $c_{0} \cdot \Phi$ for $\Phi$ restricted to the range of $c_{0}$; i.e., $c_{0} \cdot \Phi(b)=\Phi(b) \mid c_{0} \mathcal{H}_{\Phi}, b \in B$, where $\mathcal{H}_{\Phi}$ is the Hilbert space of $\Phi$. The representation $\pi_{0}$ has a unique normal extension $\pi_{0}^{\prime \prime}$ mapping $\Phi(B)^{\prime \prime}$ onto $\pi_{0}(B)^{\prime \prime}$ and the kernel of $\pi_{0}^{\prime \prime}$ is $c_{0}^{\perp}\left(\Phi(B)^{\prime \prime}\right)$. In particular, $\pi_{0}^{\prime \prime} \circ \Phi=\pi_{0}$ on $B$. (See 3.7.7 and 3.8.1 of [23].) We write $\Psi$ for the restriction of $\pi_{0}^{\prime \prime}$ to $c_{0} \Phi(B)^{\prime \prime}$, so $\Psi$ is a normal isomorphism from $c_{0} \Phi(B)^{\prime \prime}$ onto $\pi_{0}(B)^{\prime \prime}$, and $\Psi \circ\left(c_{0} \cdot \Phi\right)=$ $\pi_{0}$ on $B$. Again, by Theorem 3.7.7 of [23], there is a one-parameter group of (necessarily normal) *-automorphisms $\left\{\alpha_{t}^{\prime \prime}\right\}_{t \in \mathbf{R}}$ on $\Phi(B)^{\prime \prime}$ such that $\alpha_{t}^{\prime \prime} \circ \Phi=\Phi \circ \alpha_{t}$ on $B$ because $\Phi \circ \alpha_{t}$ is a representation of $B$. Since $\Phi(B)^{\prime \prime}$ is canonically isomorphic to the double dual of $B$ (Theorem 3.7.8 of [23]), one may view $\alpha_{t}^{\prime \prime}$ as the second transpose of $\alpha_{t}$. In general, the group $\left\{\alpha_{t}^{\prime \prime}\right\}_{t \in \mathbf{R}}$ is not continuous in any sense; that is, the map $t \rightarrow \alpha_{t}^{\prime \prime}$ is not continuous. However, $\pi_{0}^{\prime \prime} \circ \alpha_{t}^{\prime \prime}$ and $\left(A d U_{t}\right) \circ \pi_{0}^{\prime \prime}$ are normal representations of $\Phi(B)^{\prime \prime}$ that agree on $\Phi(B)$ because $\pi \circ \alpha_{t}=\left(A d U_{t}\right) \circ \pi$. Indeed, $\pi_{0}^{\prime \prime} \circ \alpha_{t}^{\prime \prime}(\Phi(b))=\pi_{0}^{\prime \prime}\left(\Phi\left(\alpha_{t}(b)\right)\right)=\pi_{0}\left(\alpha_{t}(b)\right)=\left(A d U_{t}\right) \circ \pi_{0}(b)=\left(A d U_{t}\right) \circ \pi_{0}^{\prime \prime}(\Phi(b))$. It follows that $\alpha_{t}^{\prime \prime}\left(c_{0}\right)=c_{0}$ for all $t$ and if $\tilde{\alpha}_{t}$ denotes the restriction of $\alpha_{t}^{\prime \prime}$ to $c_{0} \cdot \Phi(B)^{\prime \prime}$, then $\tilde{\alpha}_{t}=\Psi^{-1} \circ\left(A d U_{t}\right) \circ \Psi$. Consequently, $\left\{\tilde{\alpha}_{t}\right\}_{t \in \mathbf{R}}$ is an ultraweakly continuous group of automorphisms of $c_{0} \cdot \Phi(B)^{\prime \prime}$. The following theorem shows that $c_{0}$ is the largest central projection in $\Phi(B)^{\prime \prime}$ on which $\left\{\alpha_{t}^{\prime \prime}\right\}_{t \in \mathbf{R}}$ acts ultraweakly continuously. It identifies, too, conditions under which a representation is quasi-covariant. We continue using the notation already established.

Theorem 2.2. Let $\pi$ be a $C^{*}$-representation of $B$ on a separable Hilbert space and let $c=c(\pi)$ be the central support of $\pi$ in $\Phi(B)^{\prime \prime}$. Then $\pi$ is quasi-covariant if and only if $c$ is an $\left\{\alpha_{t}^{\prime \prime}\right\}_{t \in \mathbf{R}}$ invariant projection dominated by $c_{0}$.

Proof. Suppose $\pi$ is quasi-covariant. By Proposition 2.1, we may replace $\pi$ with $\infty \cdot \pi$, if necessary, in order to assume that $\pi$ is protocovariant. This does not change $c$. The invariance of $c$ under $\left\{\alpha_{t}^{\prime \prime}\right\}_{t \in \mathbf{R}}$ may be proved using exactly the same argument we used to prove that $c_{0}$ is invariant. Alternatively, one may use the equivalence of assertions (i) and (iv) of Theorem 7.4.10 in [23]. To see that $c \leq c_{0}$, choose a unitary representation $V=\left\{V_{t}\right\}_{t \in \mathbf{R}}$ of $\mathbf{R}$ on $\mathcal{H}_{\pi}$ so that $(\pi, V)$ is a covariant representation of $(B, \mathbf{R}, \alpha)$. Let $p$ be the central support of $\pi \times V$ in $\tilde{\Phi}\left(B \times{ }_{\alpha} \mathbf{R}\right)^{\prime \prime}$ and let $\tilde{\Psi}$ be a von Neumann algebra isomorphism from $p \cdot \tilde{\Phi}\left(B \times{ }_{\alpha} \mathbf{R}\right)^{\prime \prime}$ onto $(\pi \times V)\left(B \times{ }_{\alpha} \mathbf{R}\right)^{\prime \prime}$ such that $\tilde{\Psi} \circ(p \cdot \tilde{\Phi})=\pi \times V$. As shown in $\S 7.6$ of [23], $B$ lies in the multiplier algebra of $B \times{ }_{\alpha} \mathbf{R}$. Consequently, if $\left\{e_{\lambda}\right\}$ is an approximate identity for $B \times{ }_{\alpha} \mathbf{R}$ we find that for all $a \in B, \pi(a)=\lim _{\lambda}(\pi \times V)\left(a e_{\lambda}\right)=$ $\lim _{\lambda}(\tilde{\Psi} \circ(p \cdot \tilde{\Phi}))\left(a e_{\lambda}\right)=\tilde{\Psi}\left(p \cdot \lim _{\lambda}\left(\tilde{\Phi}\left(a e_{\lambda}\right)\right)\right)=\tilde{\Psi}\left(p \cdot \pi_{0}(a)\right)$, where the limit is in the ultraweak topology. Thus $\pi$ is quasi-equivalent to a subrepresentation of $\pi_{0}$, and so $c \leq c_{0}$.

For the converse, observe that for all $t, \pi \circ \alpha_{t}$ is quasi-equivalent to $c \cdot \Phi \circ \alpha_{t}=$ $c \cdot c_{0} \cdot \Phi \circ \alpha_{t}=c \cdot \tilde{\alpha}_{t} \circ\left(c_{0} \cdot \Phi\right)=\tilde{\alpha} \circ(c \cdot \Phi)$. (The last equality is justified by the assumption that $c$ is an invariant central projection dominated by $c_{0}$.) If $\Psi$ is the von Neumann algebra isomorphism from $(c \cdot \Phi)(B)^{\prime \prime}$ onto $\pi(B)^{\prime \prime}$ such that $\Psi \circ(c \cdot \Phi)=\pi$, then $\left\{\Psi \circ \tilde{\alpha}_{t} \circ \Psi^{-1}\right\}_{t \in \mathbf{R}}$ is an ultraweakly continuous group of automorphisms of $\pi(B)^{\prime \prime}$ such that $\left(\Psi \circ \tilde{\alpha}_{t} \circ \Psi^{-1}\right) \circ \pi=\pi \circ \alpha_{t}$ for all $t$. Thus, by Proposition 2.1, $\pi$ is quasi-covariant. 
We note in passing that Theorem 2.2 may also be deduced from Corollary 4.2 of [27].

Corollary 2.3. Let $\pi$ be a representation of $B$ on a separable Hilbert space $\mathcal{H}_{\pi}$. Then $\pi$ decomposes into the direct sum of two disjoint subrepresentations, $\pi=$ $\pi_{1} \oplus \pi_{2}$, where $\pi_{1}$ is quasi-covariant and $\pi_{2}$ contains no quasi-covariant subrepresentations. Further, $\pi_{2}$ decomposes into the direct sum of two disjoint subrepresentations, $\pi_{2}=\pi_{21} \oplus \pi_{22}$, where $\pi_{21}$ is contained in a quasi-covariant representation and $\pi_{22}$ is disjoint from all quasi-covariant representations.

Proof. Let $c$ be the central support of $\pi$ in $\Phi(B)^{\prime \prime}$ and let $c_{0}=c\left(\pi_{0}\right), \alpha_{t}^{\prime \prime}$, etc., be as defined as above. Define $c_{1}$ to be $c_{0} \wedge\left(\bigwedge_{t} \alpha_{t}^{\prime \prime}(c)\right)$ and set $c_{2}=I-c_{1}$. Then $c_{1}$ is the largest invariant central projection in $\Phi(B)^{\prime \prime}$ that is dominated by both $c_{0}$ and $c$. The projections $c_{1}$ and $c_{2}$ yield a decomposition of $\pi$ as the disjoint direct sum of two representations $\pi=\pi_{1} \oplus \pi_{2}$, where $\pi_{1}$ is quasi-covariant, by Theorem 2.2 and $\pi_{2}$ has no quasi-covariant summands, again, by Theorem 2.2. The projection $c_{2}$ may be written as $c_{2}=c_{21}+c_{22}$, where $c_{21}=c_{0}\left(c-\bigwedge_{t} \alpha_{t}^{\prime \prime}(c)\right)$ and $c_{22}=c\left(I-c_{0}\right)$. The corresponding representations, $\pi_{21}$ and $\pi_{22}$ have the desired properties by Theorem 2.2 .

Remark 2.4. It should be noted that no special properties of $\mathbf{R}$, beyond the fact that $\mathbf{R}$ is a 2 nd countable locally compact group, have been used so far. Thus these results remain valid for arbitrary $C^{*}$-dynamical systems $(B, G, \alpha)$ where $B$ is a separable $C^{*}$-algebra and $G$ is a second countable, locally compact group.

The following lemma is well known. However, since we lack a specific reference, we give a proof for the sake of completeness.

Lemma 2.5. If $\mathcal{A}_{1}$ and $\mathcal{A}_{2}$ are unital ultraweakly closed algebras of operators on a Hilbert space $\mathcal{H}$, then $\mathcal{A}_{1}=\mathcal{A}_{2}$ if and only if the algebras $\mathcal{A}_{1} \otimes \mathbf{C} I$ and $\mathcal{A}_{2} \otimes \mathbf{C} I$, acting on the Hilbert space $\mathcal{H} \otimes \ell^{2}(\mathbf{Z})$, have the same lattice of invariant subspaces.

Proof. Obviously the condition is necessary. To see that it is sufficient, suppose there is an $a \in \mathcal{A}_{1} \backslash \mathcal{A}_{2}$. Then there is an ultraweakly continuous linear functional $f=\sum w_{x_{i}, y_{i}}$, where $\sum\left\|x_{i}\right\|^{2}$ and $\sum\left\|y_{i}\right\|^{2}$ are finite and $w_{x_{i}, y_{i}}(T)=\left(T x_{i}, y_{i}\right)$, such that $f(a) \neq 0$ while $f(b)=0$ for all $b \in \mathcal{A}_{2}$. Viewing $\mathcal{H} \otimes \ell^{2}(\mathbf{Z})$ as the direct sum of copies of $\mathcal{H}$, let $\xi=\left(x_{i}\right)$ and $\eta=\left(y_{i}\right)$ in $\mathcal{H} \otimes \ell^{2}(\mathbf{Z})$. Then $((a \otimes I) \xi, \eta)=f(a) \neq 0=$ $f(b)=((b \otimes I) \xi, \eta)$ for all $b \in \mathcal{A}_{2}$. The space $\mathcal{K}:=\left[\left(\mathcal{A}_{2} \otimes \mathbf{C} I\right) \xi\right]$ is in $\operatorname{lat}\left(\mathcal{A}_{2} \otimes \mathbf{C} I\right)$ and $\xi \in \mathcal{K}$ because $\mathcal{A}_{2}$ is unital. What we have shown is that $\eta$ is orthogonal to $\mathcal{K}$ but not orthogonal to $(a \otimes I) \xi$. Thus, since $\xi \in \mathcal{K}, \mathcal{K} \notin \operatorname{lat}\left(\mathcal{A}_{1} \otimes \mathbf{C} I\right)$. This contradicts our hypothesis and so, by symmetry, $\mathcal{A}_{1}=\mathcal{A}_{2}$.

Recall that an algebra $\mathcal{A}$ of operators on a Hilbert space $\mathcal{H}$ is called reductive in case the invariant subspaces of $\mathcal{H}$ for $\mathcal{A}$ all reduce $\mathcal{A}$. Of course a transitive algebra is reductive, but not conversely. The reductive algebra problem is to determine whether every weakly closed reductive algebra is a von Neumann algebra. In [17], one parameter automorphism groups of von Neumann algebras were used to construct examples of ultraweakly closed reductive algebras that are not self-adjoint, i.e., not von Neumann algebras.

Corollary 2.6. An operator algebra $\mathcal{A}$ on a Hilbert space $\mathcal{H}$ is ultraweakly dense in the von Neumann algebra that it generates if and only if $\mathcal{A} \otimes \mathbf{C} I$, acting on $\mathcal{H} \otimes \ell^{2}(\mathbf{Z})$, is reductive. 
Proof. This is immediate from Lemma 2.5, the double commutant theorem, and the fact the invariant subspaces for a von Neumann algebra are the ranges of the projections in its commutant.

Returning to our $C^{*}$-dynamical system, $(B, \mathbf{R}, \alpha)$, and the analytic subalgebra $A$, we apply the results obtained so far, coupled with [17], to analyze the invariant subspace lattice of $\pi(A)$, where $\pi$ is a $C^{*}$-representation of $B$. We assume that there are no $\alpha$-invariant ideals $\mathcal{I}$ in $B$ such that the induced action on $B \backslash \mathcal{I}$ is trivial. This implies that for each $C^{*}$-representation $\pi$ of $B, \pi(A)$ is a proper, norm closed, subalgebra of $\pi(B)$.

Theorem 2.7. If $\pi$ is a $C^{*}$-representation of $B$, then $\pi(A)$ has a non-reducing invariant subspace if and only if $\pi$ contains a protocovariant subrepresentation.

Proof. For the sufficiency of the condition, observe that if $\pi$ contains a protocovariant subrepresentation, we may as well assume that $\pi$, itself, is protocovariant. If $\left\{U_{t}\right\}_{t \in \mathbf{R}}$ is a unitary representation of $\mathbf{R}$ such that $(\pi, U)$ is a covariant representation of $(B, \mathbf{R}, \alpha)$, and if $E$ is the spectral measure for $U$, then by Theorem 2.9 of [17] and the fact that $\left(A d U_{t}\right) \circ \pi=\pi \circ \alpha_{t}$, each of the subspaces $E([t, \infty)) \mathcal{H}_{\pi}$ is invariant under $\pi(A)$. They can't all reduce $\pi(A)$ because if they did, they would reduce $\pi(B)$. This and the covariance assumption, then, would imply that $\operatorname{ker} \pi$ is invariant and that $\alpha$ is trivial on $B / \operatorname{ker} \pi$ contrary to our assumptions.

For the converse, suppose $\mathcal{M}$ is an invariant subspace for $\pi(A)$ that does not reduce $\pi(A)$. For each $t \in \mathbf{R}$, let $\widetilde{\mathcal{M}}_{t}$ be the closure of $\pi\left(B^{\alpha}[t, \infty)\right) \mathcal{M}$ where, recall, $B^{\alpha}[t, \infty)$ is the spectral subspace of $B$ determined by $\alpha$ and the interval $[t, \infty)$. (See Chapter 8 of [23].) Let $\mathcal{M}_{\infty}=\bigwedge_{t \in \mathbf{R}} \widetilde{\mathcal{M}}_{t}$ and let $\mathcal{M}_{-\infty}=\bigvee_{t \in \mathbf{R}} \widetilde{\mathcal{M}}_{t}$ The spaces $\mathcal{M}_{ \pm \infty}$ reduce $\pi(A)$, as is shown in the proof of Theorem 5.2 of [17]. Furthermore, $\mathcal{N}:=\mathcal{M}_{-\infty} \ominus \mathcal{M}_{\infty}$ is non-zero by our assumption that $\mathcal{M}$ does not reduce $\pi(A)$ and if $\mathcal{N}_{t}=\mathcal{N} \wedge\left(\bigwedge_{s<t} \widetilde{\mathcal{M}}_{s}\right)$, then there is a spectral measure $E$ on $\mathbf{R}$ with values in the projections on $\mathcal{N}$ such that $\mathcal{N}_{t}=E[t, \infty) \mathcal{N}$ for all $t$ and for each $t$ and $s$ in $\mathbf{R}, \pi\left(B^{\alpha}[t, \infty)\right) E[s, \infty) \mathcal{N} \subseteq E[t+s, \infty) \mathcal{N}$. If $U=\left\{U_{t}\right\}_{t \in \mathbf{R}}$ is the Fourier-Stieltjes transform of $E$ and if $\pi_{\mathcal{N}}$ is the reduction of $\pi$ by $\mathcal{N}$, then $\pi_{\mathcal{N}}$ is a subrepresentation of $\pi$ and by Theorem 2.13 of [17], $\left(\pi_{\mathcal{N}}, U\right)$ is a covariant representation of $(B, \mathbf{R}, \alpha)$.

Corollary 2.8. If $\pi$ is a $C^{*}$-representation of $B$, then $\pi(A)$ is ultraweakly dense in $\pi(B)^{\prime \prime}$ if and only if $\pi$ contains no quasi-covariant subrepresentations.

Proof. By Corollary 2.6, $\pi(A)$ is ultraweakly dense in $\pi(B)^{\prime \prime}$ (the von Neumann algebra generated by $\pi(A))$ if and only if $(\infty \cdot \pi)(A)$ is a reductive algebra. By Theorem 2.7, this happens if and only if $\infty \cdot \pi$ contains no protocovariant representations. If $\sigma$ is a quasi-covariant representation of $\pi$, then $\infty \cdot \sigma$ is a protocovariant subrepresentation of $\infty \cdot \pi$ by Proposition 2.1. So $\pi(A)$ is not ultraweakly dense in $\pi(B)^{\prime \prime}$. On the other hand, if $\pi(A)$ is not ultraweakly dense, then $\infty \cdot \pi$ contains a protocovariant representation $\sigma$. Since $\pi$ is quasi-equivalent to $\infty \cdot \pi, \sigma$ is quasi-equivalent to a subrepresentation of $\pi$. Since quasi-equivalence preserves quasi-covariance, we conclude that $\pi$ contains a quasi-covariant representation.

Proof of Theorem 1.1. If $\pi$ is an irreducible representation of $B$, then $\pi$ contains no proper subrepresentations. So, to show that $\pi(A)$ is ultraweakly dense in $\mathcal{L}(\mathcal{H})$, it suffices, by Corollary 2.8, to show that $\pi$ is not quasi-covariant. If $\pi$ were 
quasi-covariant, then there would be an ultraweakly continuous representation of $\mathbf{R}$ $\left\{\beta_{t}\right\}_{t \in \mathbf{R}}$ on $\pi(B)^{\prime \prime}=\mathcal{L}\left(\mathcal{H}_{\pi}\right)$ such that $\beta_{t} \circ \pi=\pi \circ \alpha_{t}$ for all $t$. It is well known that an ultraweakly continuous representation of $\mathbf{R}$ on $\mathcal{L}\left(\mathcal{H}_{\pi}\right)$ is spatially implemented. Thus there is a unitary representation $\left\{U_{t}\right\}_{t \in \mathbf{R}}$ of $\mathbf{R}$ on $\mathcal{H}_{\pi}$ such that $\beta_{t}=A d U_{t}$, and so $\pi$ is, in fact, protocovariant. Thus by Theorem 2.7, $\pi(A)$ has a proper invariant subspace, contrary to assumption. Thus $\pi$ cannot be quasi-covariant.

The next result is the key to proving Theorem 1.2. It assumes something a bit weaker than strong Dirichlet property on $A$. This will prove useful later.

Theorem 2.9. Suppose $\pi$ is a quasi-covariant representation of $B$ on the Hilbert space $\mathcal{H}_{\pi}$. Let $M=\pi(B)^{\prime \prime}$ and let $\left\{\beta_{t}\right\}_{t \in \mathbf{R}}$ be the ultraweakly continuous representation of $\mathbf{R}$ on $M$, guaranteed by Proposition 2.1, such that $\beta_{t} \circ \pi=\pi \circ \alpha_{t}$ for all $t \in \mathbf{R}$. If $\pi\left(B^{\alpha}(-\infty, 0)\right)+\pi\left(B^{\alpha}(0)\right)+\pi\left(B^{\alpha}(0, \infty)\right)$ is ultraweakly dense in $M$, in particular, if $A$ is a strongly Dirichlet subalgebra of $B$, then $\pi(A)$ is ultraweakly dense in $M^{\beta}[0, \infty)$, where $M^{\beta}[0, \infty)$ is the spectral subspace of $M$ determined by $\beta$ and the interval $[0, \infty)$.

Proof. Since $\beta_{t} \circ \pi=\pi \circ \alpha_{t}, \pi(A) \subseteq M^{\beta}[0, \infty)$. If $\pi(A)$ is not ultraweakly dense in $M^{\beta}[0, \infty)$, we may apply Lemma 2.5 , replace $\pi$ by $\infty \cdot \pi$, if necessary, and suppose that there is a subspace $\mathcal{M}$ of $\mathcal{H}_{\pi}$ that is invariant under $\pi(A)$ but not under $M^{\beta}[0, \infty)$. Of course $\mathcal{M}$ does not reduce $\pi$. So by Theorem $2.7, \pi$ contains a protocovariant representation say $\rho$. The subspace carrying $\rho$ reduces $\pi$, and therefore $M$, and so we may cut down to it, if necessary, and assume that $\pi$, itself, is protocovariant. Reviewing the proof of Theorem 2.7, which follows arguments in [17], we see that there is a unitary representation $V=\left\{V_{t}\right\}_{t \in \mathbf{R}}$ of $\mathbf{R}$ on $\mathcal{H}_{\pi}$ with spectral measure $E$ such that $\left(A d V_{t}\right) \circ \pi=\pi \circ \alpha_{t}$ for all $t$ (so that, in fact, $\left.\beta_{t}=A d V_{t}\right)$ and such that for all $\epsilon>0$

$$
E[\epsilon, \infty) \leq Q \leq E[0, \infty)
$$

where $Q$ is the projection of $\mathcal{H}_{\pi}$ onto $\mathcal{M}$. Thus $E(0, \infty) \leq Q \leq E[0, \infty)$. We shall write $E_{0}$ for $E\{0\}=E[0, \infty)-E(0, \infty)$. Note that $E_{0}$ is the projection onto fixed vectors for $\left\{V_{t}\right\}_{t \in \mathbf{R}}$. (This projection may, a priori, be zero. But if it is, then $E(0, \infty)=Q=E[0, \infty)$ and $\mathcal{M}$ is invariant under $M^{\beta}[0, \infty)$ by Corollary 2.14 of [17]. Since this contradicts our assumption, we shall proceed under the assumption that $E_{0} \neq 0$.) By Corollary 2.14 of [17], we have

$$
E_{0} \pi\left(B^{\alpha}(0, \infty)\right) E_{0} \subseteq E_{0} M^{\beta}(0, \infty) E_{0}=0,
$$

where $M^{\beta}(0, \infty)$ is the ultraweak closure of $\left\{T \in M \mid s p_{\beta}(T)\right.$ is compact and contained in $(0, \infty)\}$. Therefore $E_{0} \pi\left(B^{\alpha}(-\infty, 0)\right) E_{0} \subseteq E_{0} M^{\beta}(-\infty, 0) E_{0}=\{0\}$, too. Therefore, by our hypothesis that $\pi\left(B^{\alpha}(-\infty, 0)\right)+\pi\left(B^{\alpha}(0)\right)+\pi\left(B^{\alpha}(0, \infty)\right)$ is ultraweakly dense in $M$, we conclude that

$$
E_{0} M E_{0}=E_{0} \pi(B)^{\prime \prime} E_{0}=E_{0} \pi\left(B^{\alpha}(0)\right)^{\prime \prime} E_{0} .
$$

If $a \in M^{\beta}[0, \infty)$, then since $E(0, \infty)$ and $E[0, \infty)$ are invariant projections for $a$, by Corollary 2.14 of $[17]$, we have $(I-E(0, \infty)) a(E(0, \infty))=(I-E[0, \infty)) a(E[0, \infty))=$ 0 and so

$(I-Q) a Q=(E[0, \infty)-Q) a(Q-E(0, \infty))=(E[0, \infty)-Q) E_{0} a E_{0}(Q-E(0, \infty))$.

But $E_{0} a E_{0}$ lies in $\pi\left(B^{\alpha}(0)\right)^{\prime \prime}$, as we just saw, and $\pi\left(B^{\alpha}(0)\right)^{\prime \prime}$ commutes $Q$ (because $\mathcal{M}$ is invariant for $\left.\pi\left(B^{\alpha}(0)\right)\right)$ and with the spectral measure $E$ (because $A d V_{t} \circ \pi=$ 
$\pi \circ \alpha_{t}$ and $B^{\alpha}(0)$ is the fixed point algebra of $\alpha$ ). Thus we conclude that the last term in the equation is zero, so $(I-Q) a Q=0$. This contradicts our hypothesis that $\mathcal{M}$ is not invariant for $M^{\alpha}[0, \infty)$ and completes the proof.

Proof of Theorem 1.2. Suppose $\pi$ is an irreducible representation of $B$. If $\pi(A)$ has no nontrivial invariant subspaces, then, as we have noted before, Theorem 1.2 follows from Theorem 1.1. On the other hand, if $\pi(A)$ has a nontrivial invariant subspace, then $\pi$ has protocovariant subrepresentation by Theorem 2.7. Since $\pi$ is assumed to be irreducible, $\pi$ must be protocovariant, say $\left(A d V_{t}\right) \circ \pi=\pi \circ \alpha_{t}$ for some unitary representation $V=\left\{V_{t}\right\}_{t \in \mathbf{R}}$ of $\mathbf{R}$ on $\mathcal{H}_{\pi}$. Also, since $\pi$ is irreducible, $M:=\pi(B)^{\prime \prime}$ is $\mathcal{L}\left(\mathcal{H}_{\pi}\right)$. So if $\beta_{t}=A d V_{t}, \pi(A)$ is ultraweakly dense in $M^{\beta}[0, \infty)$, by Theorem 2.9. By Corollary 4.2.4 of [17] (and its proof), $M^{\beta}[0, \infty)=\operatorname{alg}(\mathcal{N})$, where $\mathcal{N}$ is the nest $\left\{E[t, \infty) \mathcal{H}_{\pi}\right\}_{t \in \mathbf{R}}$ and $E$ is the spectral measure of $\left\{V_{t}\right\}_{t \in \mathbf{R}}$.

\section{Uniform AVERAGing AND the KAFtal-LARson-Weiss DENSity theOREM}

To begin this section, we investigate some of the relations among the uniform averaging condition, the strong Dirichlet hypothesis, and the ergodic theorem of Dang-Ngoc-Nghiem [7]. Our particular objective here is to repair an error in [13]. Our analysis will help to clarify the hypothesis in Theorem 1.2 and to provide a new proof of Theorem 10 of [15]. In fact, we shall generalize this result somewhat.

We start with a lemma that paraphrases Théorème 2.1 and Théorème 3.1 of [7]. In it, $\mathcal{B}$ will be an arbitrary von Neumann algebra and $\beta=\left\{\beta_{t}\right\}_{t \in \mathbf{R}}$ will be a 1-parameter group of automorphisms of $\mathcal{B}$. We do not suppose that the map $t \rightarrow \beta_{t}$, from $\mathbf{R}$ to $\operatorname{Aut}(\mathcal{B})$, is continuous in any sense. We write $\mathcal{D}$ for the fixed point algebra of $\beta$. Of course we shall be interested primarily in the case when $\mathcal{B}=\Phi(B)^{\prime \prime}, \beta=\alpha^{\prime \prime}$, and $\mathcal{D}=D$ - the fixed point algebra of $\alpha^{\prime \prime}$.

Lemma 3.1. With the notation just established, we have the following assertions:

(A) There is a normal, positive, linear, idempotent map $E$ on $\mathcal{B}$ whose range is contained in $\mathcal{D}$ such that

(Ai) $E\left(d_{1} b d_{2}\right)=d_{1} E(b) d_{2}$, for all $b \in \mathcal{B}$ and $d_{1}, d_{2} \in \mathcal{D}$;

(Aii) $E \circ \beta_{t}=E$ for all $t \in \mathbf{R}$;

(Aiii) If $f$ is an invariant linear functional in the predual of $\mathcal{B}$, then $f \circ E=f$.

(B) The projection $e_{0}:=E(I)$ (which lies in $\mathcal{D}$ by $(\mathrm{Ai})$ ) is the span $\bigvee s(\varphi)$, taken over all invariant normal states $\varphi$ on $\mathcal{B}$, of the support projections $s(\varphi)$ of $\varphi$.

(C) If $E^{\prime}: \mathcal{B} \rightarrow \mathcal{D}$ is also a normal, positive, linear idempotent map satisfying (Ai) and (Aii), then $e^{\prime}:=E^{\prime}(I)$ satisfies $e^{\prime} \leq e_{0}$ and $E^{\prime}(b)=e^{\prime} E(b)=E(b) e^{\prime}$ for all $b \in \mathcal{B}$. In particular, $E$ is uniquely determined by $(\mathrm{Ai})$, (Aii) and (Aiii). If $e_{0}=I$, so $\mathcal{B}$ has a separating family of $\beta$-invariant normal states, then $E$ is uniquely determined by (Ai) and (Aii).

Lemma 3.1 is really a paraphrase of Théorème 3.1 of [7]. Part B is presented to highlight the role of Théorème 2.1 of [7] in our considerations.

The projection $e_{0}$ is called the $\beta$-finite projection in $\mathcal{D}$ and the system $(\mathcal{B}, \beta)$ is called $\beta$-finite if $e_{0}=I$.

The fixed point algebra of $\alpha^{\prime \prime}, D$, is a von Neumann subalgebra of $\Phi(B)^{\prime \prime}$ that contains the ultraweak closure of $\Phi\left(B^{\alpha}(0)\right)$ because $\alpha_{t}^{\prime \prime} \circ \Phi=\Phi \circ \alpha_{t}$. However, the latter can be quite a bit smaller. Indeed, if $B$ is the compact operators on an infinite dimensional Hilbert space $\mathcal{H}$ and if $\alpha_{t}=A d U_{t}$, where $\left\{U_{t}\right\}_{t \in \mathbf{R}}$ has continuous spectrum (say, $\left\{U_{t}\right\}_{t \in \mathbf{R}}$ is translation on $L^{2}(\mathbf{R})$ ), then $B^{\alpha}(0)=\{0\}$, 
$\Phi(B)^{\prime \prime}=\mathcal{L}(\mathcal{H})$, and $D=\left(\left\{U_{t}\right\}_{t \in \mathbf{R}}\right)^{\prime}$. In Theorem 3.4, we shall prove that $A$ is a strongly Dirichlet subalgebra of $B$ if and only if the $\alpha^{\prime \prime}$-finite part of $D, e_{0} D$, where $e_{0}$ now is the $\alpha^{\prime \prime}$-finite projection in $D$, is contained in the ultraweak closure of $c \Phi\left(B^{\alpha}(0)\right)$, where $c$ is the central cover of $e_{0}$ calculated in the center of $\Phi(B)^{\prime \prime}$. This should be compared with Corollary 6.3 of [13] which, in turn, rests on part (a) of Theorem 6.1 of [13], where Jorgensen asserts that if $B$ is unital, then $\left(\Phi(B)^{\prime \prime}, \alpha^{\prime \prime}\right)$ is $\alpha^{\prime \prime}$-finite. That assertion, however, is incorrect, as the following example shows.

Example. Let $X$ be the one point compactification of $\mathbf{R}, X=\mathbf{R} \cup\{\infty\}$, and let $\mathbf{R}$ act on $X$ by translation, fixing $\infty$. Let $B=C(X)$ and let $\alpha$ be given by the action of $\mathbf{R}$ on $X$. Then $\Phi(B)^{\prime \prime}$ is the $\ell^{\infty}$-direct sum of the spaces $L^{\infty}(X, \mu)$ where $\mu$ ranges over all probability measures on $X$. These $\mu$ are, of course, the states on $B$ and the normal states on $\Phi(B)^{\prime \prime}$. Now there is only one invariant probability measure on $X$, the point mass at infinity, $\delta_{\infty}$. It follows from Lemma 3.1 that the projection $e_{0}$ has rank 1 and so $\left(\Phi(B)^{\prime \prime}, \alpha^{\prime \prime}\right)$ cannot be $\alpha^{\prime \prime}$-finite.

To prove Theorem 3.4, we require two lemmas.

Lemma 3.2. In the notation of $\S 2, e_{0} \leq c_{0}$.

Proof. By part B of Lemma 3.1, applied to the system $\left(\Phi(B)^{\prime \prime}, \alpha^{\prime \prime}\right)$, we need only show that $s(\varphi) \leq c_{0}$ for each invariant normal state $\varphi$ on $\Phi(B)^{\prime \prime}$. We view these interchangeably as invariant states on $B$. Recall that if $N_{\varphi}=\left\{b \in \Phi(B)^{\prime \prime}\right.$ $\left.\varphi\left(b^{*} b\right)=0\right\}$, then $N_{\varphi}$ is an ultraweakly closed left ideal in $\Phi(B)^{\prime \prime}$ and is expressed as $N_{\varphi}=\Phi(B)^{\prime \prime}(1-s(\varphi))$. (See $\left[8\right.$, I.4.6].) On the other hand, if $\pi_{\varphi}$ is the representation of $\Phi(B)^{\prime \prime}$ determined by $\varphi$ through the GNS construction, then $\operatorname{ker} \pi_{\varphi}=$ $\Phi(B)^{\prime \prime}\left(1-c_{\varphi}\right)$, where $c_{\varphi}$ is the support of $\pi_{\varphi}$, a projection in the center of $\Phi(B)^{\prime \prime}$. However, $\operatorname{ker} \pi_{\varphi}$ is contained in $N_{\varphi}$ and so $s(\varphi) \leq c_{\varphi}$. Since $\varphi$ is an invariant state on $B$ and since, as is well known, the GNS representations associated to invariant states are protocovariant, we conclude that $c_{\varphi} \leq c_{0}$ by Theorem 2.2. Thus $s(\varphi) \leq c_{0}$

The second lemma gives three other conditions that are all equivalent to the condition that $A$ is a strongly Dirichlet subalgebra of $B$. In Theorem 3.1 of [20] these were claimed to be equivalent to a fourth condition. However, the proof given there rests on part (a) of Jorgensen's Theorem 6.1 in [13] and as it turns out we had to drop the fourth condition and provide new arguments. We did this in our first draft. However, László Zsidó then called our attention to [27, Corollary 3.5] where the lemma is proved almost exactly as we need it. In the lemma we shall write $B^{\prime}$ for the Banach space dual of $B$. (Recall that we are identifying $\Phi(B)^{\prime \prime}$ with the double dual of $B$, so this notation is consistent.) Also, given a map $\Psi: B \mapsto B$, we denote the transpose or adjoint of $\Psi$ mapping $B^{\prime}$ to $B^{\prime}$ by $\Psi^{\prime}$. Finally, we shall write $\left(B^{\prime}\right)^{\alpha}(0)$ for the invariant elements of $B^{\prime}$.

Lemma 3.3. The following assertions are equivalent:

(1) There is an $\alpha$ invariant conditional expectation $\Psi$ from $B$ onto $B^{\alpha}(0)$ such that, for all $b \in B$,

$$
\lim _{T \longrightarrow \infty} \frac{1}{2 T} \int_{-T}^{T} \alpha_{t}(b) d t=\Psi(b)
$$

in norm. 
(2) There is an $\alpha$ invariant conditional expectation $\Psi$ from $B$ onto $B^{\alpha}(0)$ such that, for all $b \in B$,

$$
\lim _{y \longrightarrow \infty} \int_{-\infty}^{\infty} \alpha_{t}(b) P_{y}(t) d t=\Psi(b)
$$

in norm, where $P_{y}(t)=\frac{1}{\pi} \frac{y}{y^{2}+t^{2}}$ is the Poisson kernel.

(3) There is an $\alpha$ invariant expectation $\Psi$ from $B$ onto $B^{\alpha}(0)$ such that $\Psi^{\prime}: B^{\prime} \mapsto$ $B^{\prime}$ maps onto $\left(B^{\prime}\right)^{\alpha}(0)$.

(4) The algebra $A$ is a strongly Dirichlet subalgebra of $B$.

Proof. As we said above, this is essentially Corollary 3.5 of [27]. However, one needs to cite his Lemma 3.3 for the equivalence of the first two conditions. Also, his corollary is phrased in terms of one parameter groups of operators on a Banach space. The fact that the projection his lemma guarantees is an expectation in our setting is, fortunately, quite elementary.

Theorem 3.4. The algebra $A$ is a strongly Dirichlet subalgebra of $B$ if and only if $e_{0} D$ coincides with the ultraweak closure of $c \cdot \Phi\left(B^{\alpha}(0)\right)$, where $c$ is the central cover of $e_{0}$ in the center of $\Phi(B)^{\prime \prime}$.

We suspect that $c$ lies in the ultraweak closure of $\Phi\left(B^{\alpha}(0)\right)$, so the conclusion may be rephrased to say that $e_{0} D$ is contained in the ultraweak closure of $\Phi\left(B^{\alpha}(0)\right)$, but we are unable to prove this.

Proof. Suppose that $A$ is a strongly Dirichlet subalgebra of $B$ and recall that $\Phi(B)^{\prime \prime}$ is canonically isometrically isomorphic to the Banach space double dual of $B$. By 3.3 there is a faithful, $\alpha$-invariant, conditional expectation $\Psi$ from $B$ onto $B^{\alpha}(0)$ such that $\Psi^{\prime}$ on $B^{\prime}$ maps onto $\left(B^{\prime}\right)^{\alpha}(0)$, where, recall, $B^{\prime}$ denotes the Banach space dual of $B,\left(B^{\prime}\right)^{\alpha}(0)$ denotes the invariant functionals on $B$ and $\Psi^{\prime}$ denotes the transposed or adjoint map on $B^{\prime}$. (Note that $\left(B^{\prime}\right)^{\alpha}(0)$ is the space of functionals whose spectra, calculated with respect to the transposed action of $\mathbf{R}$ on $B^{\prime}$ determined by $\alpha$, are contained in $\{0\}$; cf. [17, Lemma 2.2].) The second transpose or adjoint of $\Psi, \Psi^{\prime \prime}$, then is a normal, idempotent linear map on $\Phi(B)^{\prime \prime}$. But also, $\Psi^{\prime \prime}$ is positive because $\Psi$ is positive. Next observe that the range of $\Psi^{\prime \prime}$ is contained in $D$. To see this, denote the pairing between $\Phi(B)^{\prime \prime}$ and $B^{\prime}$ by $\langle$,$\rangle . Then for all b \in \Phi(B)^{\prime \prime}$, and $f \in B^{\prime}\left\langle\alpha_{t}^{\prime \prime}\left(\Psi^{\prime \prime}(b)\right), f\right\rangle=\left\langle\Psi^{\prime \prime}(b), \alpha_{t}^{\prime}(f)\right\rangle=\left\langle b, \Psi^{\prime}\left(\alpha_{t}^{\prime}(f)\right)\right\rangle=\left\langle b, \Psi^{\prime}(f)\right\rangle=\left\langle\Psi^{\prime \prime}(b), f\right\rangle$, showing that $\alpha_{t}^{\prime \prime}\left(\Psi^{\prime \prime}(b)\right)=\Psi^{\prime \prime}(b)$, that is, that $\Psi^{\prime \prime}\left(\Phi(B)^{\prime \prime}\right) \subseteq D$. To see that $\Psi^{\prime \prime}$ satisfies condition (Ai) in Lemma 3.1, observe that if $b \in \Phi(B)^{\prime \prime}$ and $d_{1}, d_{2} \in D$, then for all $f \in B^{\prime}$,

$$
\begin{aligned}
\left\langle\Psi^{\prime \prime}\left(d_{1} b d_{2}\right)-d_{1} \Psi^{\prime \prime}(b) d_{2}, f\right\rangle & =\left\langle d_{1} b d_{2}-d_{1} \Psi^{\prime \prime}(b) d_{2}, \Psi^{\prime}(f)\right\rangle \\
& =\left\langle b-\Psi^{\prime \prime}(b), d_{2} \cdot \Psi^{\prime}(f) d_{1}\right\rangle .
\end{aligned}
$$

Here, we treat $B^{\prime}$ as a bimodule over $\Phi(B)^{\prime \prime}$ in the usual way (see [8, Part I, Chapter $4, \S 7])$. However, $d_{2} \Psi^{\prime}(f) d_{1}$ is an invariant normal linear functional on $\Phi(B)^{\prime \prime}$ and therefore an invariant element of $B^{\prime}$. Since the range of $\Psi^{\prime}$ on $B^{\prime}$ is the space of all invariant linear functionals on $B$, we may write $d_{2} \Psi^{\prime}(f) d_{1}=\Psi^{\prime}(g)$ for some $g \in B^{\prime}$. The last term in equation 3.1 then becomes $\left\langle b-\Psi^{\prime \prime}(b), \Psi^{\prime}(g)\right\rangle=$ $\left\langle\Psi^{\prime \prime}(b)-\left(\Psi^{\prime \prime}\right)^{2}(b), g\right\rangle=0$. Condition (Aii) in Lemma 3.1 is also satisfied because of duality. Finally, if $f$ is an invariant normal linear functional on $\Phi^{\prime \prime}(B)$, then for all $b \in \Phi(B)^{\prime \prime}, f \circ\left(\Psi^{\prime \prime}(b)\right)=\left\langle\Psi^{\prime \prime}(b), f\right\rangle=\left\langle b, \Psi^{\prime}(f)\right\rangle=\langle b, f\rangle=f(b)$; i.e., $f \circ \Psi^{\prime \prime}=f$. Thus, by Lemma 3.1, $\Psi^{\prime \prime}$ is the map $E$ described there. 
Recall that $c$ is the central cover of $e_{0}$ in the center of $\Phi(B)^{\prime \prime}$. Evidently, in the notation of Lemma 3.2, $c=\bigvee c_{\varphi}$. By Lemma 3.2, $c \leq c_{0}$. Observe, too, that $\Psi^{\prime \prime}=\Psi_{c}^{\prime \prime}$, where $\Psi_{c}^{\prime \prime}(b)=\Psi^{\prime \prime}(c b c)=\Psi^{\prime \prime}(c b)$ for all $b \in \Phi(B)^{\prime \prime}$.

Now set $\Phi_{c}(b)=\Phi(b) c=c \Phi(b), b \in B$. Then $\Phi_{c}$ may be viewed as the restriction of $\Phi$ to the range of $c$. Because $c \leq c_{0}$, we have $\Phi_{c} \circ \alpha_{t}=\tilde{\alpha}_{t} \circ \Phi_{c}$, where, recall, $\tilde{\alpha}_{t}$ is $\alpha_{t}^{\prime \prime}$ restricted to $c_{0} \Phi(B)^{\prime \prime}$ - this is an ultraweakly continuous representation of R. If we define $\tilde{\Psi}_{y}, y>0$, on $c \Phi(B)^{\prime \prime}$ by the formula, $\tilde{\Psi}_{y}(b)=\int_{-\infty}^{\infty} \tilde{\alpha}_{t}(b) P_{y}(t) d t$, then it is evident that $\tilde{\Psi}_{y} \circ \Phi_{c}=\Phi_{c} \circ \Psi_{y}$ for all positive $y$. This formula and Kaplansky's density theorem imply that the $\tilde{\Psi}_{y}$ tend pointwise ultraweakly to a positive linear map $\tilde{\Psi}$ on $c \Phi(B)^{\prime \prime}$ such that $\tilde{\Psi} \circ \Phi_{c}=\Phi_{c} \circ \Psi$. We extend $\tilde{\Psi}$ to all of $\Phi(B)^{\prime \prime}$ by setting it equal to zero on $(1-c) \Phi(B)^{\prime \prime}$. Then it is straightforward to verify that $\tilde{\Psi}$ is a normal, positive linear, idempotent map from $\Phi(B)^{\prime \prime}$ into $D$. Condition (Ai) of Lemma 3.1 is satisfied because the $\tilde{\Psi}_{y}$ clearly satisfy (Ai). Condition (Aii) of Lemma 3.1 follows easily from the fact that $\tilde{\Psi} \circ \Phi_{c}=\Phi_{c} \circ \Psi$, the ultraweak density of $\Phi_{c}(B)$ in $\Phi_{c}(B)^{\prime \prime}$, and the invariance of $\Psi$ under $\left\{\alpha_{t}\right\}_{t \in \mathbf{R}}$. Finally, condition (Aiii) follows from the fact that if $f$ is an invariant element of $B^{\prime}$, then $f \circ \tilde{\Psi}_{y}=f$ for all $y$. We conclude from the uniqueness assertion in Lemma 3.1 that $\tilde{\Psi}=E$, also. That is, $\tilde{\Psi}=\Psi^{\prime \prime}$. Thus we have shown that $\Psi^{\prime \prime} \circ \Phi_{c}=\Phi_{c} \circ \Psi$. (Alternatively, this equation follows by duality as follows: for all $f \in \Phi(B)^{\prime}$, and $b \in B$, we have $\left\langle\Psi^{\prime \prime}\left(\Phi_{c}(b)\right), f\right\rangle=\left\langle\Phi_{c}(b), \Psi^{\prime}(f)\right\rangle=\left\langle\Phi(b), \Psi^{\prime}(f)\right\rangle$ because $c$ dominates the support of $f$. Therefore, the last term is the same as $\left\langle b, \Psi^{\prime}(f)\right\rangle=\langle\Psi(b), f\rangle=\langle\Phi(\Psi(b)), f\rangle=\left\langle\Phi_{c}(\Psi(b)), f\right\rangle$, where the last equation is justified by the fact that the range of $\Psi$ on $B$ consists of invariant elements. Since these equations hold for all $f \in \Phi(B)$, the result follows.)

Now by (Ai) of Lemma 3.1 we may write $e_{0} D=\Psi^{\prime \prime}\left(\Phi(B)^{\prime \prime}\right)$. But since $\Psi^{\prime \prime}=\Psi_{c}^{\prime \prime}$, $\Psi^{\prime \prime}\left(\Phi(B)^{\prime \prime}\right)={\overline{\Psi^{\prime \prime}\left(\Phi_{c}(B)\right)}}^{u w}$. Since $\Psi^{\prime \prime} \circ \Phi_{c}=\Phi_{c} \circ \Psi$, this last space is $\overline{\Phi_{c}(\Psi(B))}$ uw , which in turn is ${\bar{\Phi}\left(B^{\alpha}(0)\right)}^{u w}$. That is, we have shown that $e_{0} D=c \cdot \overline{\Phi\left(B^{\alpha}(0)\right)} u$.

For the converse assertion, suppose that $f \in B^{\prime}$ annihilates $B^{\alpha}(-\infty, 0)+B^{\alpha}(0)+$ $B^{\alpha}(0, \infty)$. Then by Proposition 5.1 of [3], $f$ is invariant. By Lemma 3.1 of $[7], f$ is supported by $c \Phi(B)^{\prime \prime}$; i.e., $c f=f c=f$. By condition (Aiii) of Lemma 3.1 and the fact that $\Psi^{\prime \prime}=E$, we conclude that $f \circ \Psi^{\prime \prime}=f$. By hypothesis the range of $\Psi^{\prime \prime}$, which is $e_{0} D$, is contained in $c{\overline{\Phi\left(B^{\alpha}(0)\right.}}^{u w}$. Since $f \cdot c=f$ and since $f$ annihilates $B^{\alpha}(0)$, we conclude that $f=0$.

The following corollary of the proof of the converse part of Theorem 3.4 deserves to be singled out. It complements Theorem 2.9 and Theorem 1.2.

Corollary 3.5. Suppose that $\pi$ is a quasi-covariant representation of $B$ on the Hilbert space $\mathcal{H}_{\pi}$. Let $M=\pi(B)^{\prime \prime}$ and let $\beta=\left\{\beta_{t}\right\}_{t \in \mathbf{R}}$ be the ultraweakly continuous representation of $\mathbf{R}$ on $M$ such that $\beta_{t} \circ \pi=\pi \circ \alpha_{t}$. Also, let $e_{0}$ be the $\beta$-finite projection for the system $(M, \beta)$ and let $c$ be the central cover of $e_{0}$ in the center of $M$. If $e_{0} M^{\beta}(0)$ is contained in the ultraweak closure of $c \cdot \pi\left(B^{\alpha}(0)\right)$, then $\pi(A)$ is ultraweakly dense in $M^{\beta}[0, \infty)$.

Proof. By Theorem 2.9, we must show that $\pi\left(B^{\alpha}(-\infty, 0)\right)+\pi\left(B^{\alpha}(0)\right)+\pi\left(B^{\alpha}(0, \infty)\right)$ is ultraweakly dense in $M$. If $f$ is an element in the predual of $M$ that annihilates this space, then by Proposition 5.1 of [3], $f$ is invariant. Therefore $f=f \circ E$ where $E: M \rightarrow M^{\beta}(0)$ is the map of Lemma 3.1. The range of $E$ is $e_{0} M^{\beta}(0)$ which, by 
hypothesis, is contained in $c \cdot \pi\left(B^{\alpha}(0)\right)$. Since $f \cdot c=c \cdot f=f$ and $f$ annihilates $\pi\left(B^{\alpha}(0)\right), f$ annihilates $c \cdot \pi\left(B^{\alpha}(0)\right)$, too. Thus $f=0$.

The next result is our way of approaching the Kaftal-Larson-Weiss theorem [15, Theorem 10].

Theorem 3.6. Suppose that $M$ is a semifinite von Neumann algebra on a separable Hilbert space and let $\mathcal{K}$ be an ultraweakly dense 2-sided ideal in $M$. Suppose that $\alpha=\left\{\alpha_{t}\right\}_{t \in \mathbf{R}}$ is an ultraweakly continuous representation of $\mathbf{R}$ in the automorphisms of $M$ satisfying the following two conditions:

(i) $\alpha$ preserves a faithful, normal, semifinite trace $\tau$, and

(ii) on $\mathcal{K}, \alpha$ is strongly continuous, i.e., $\lim _{t \rightarrow 0}\left\|\alpha_{t}(a)-a\right\|=0$ for all $a \in \mathcal{K}$.

Then $M^{\alpha}[0, \infty) \cap \mathcal{K}$ is ultraweakly dense in $M^{\alpha}[0, \infty)$.

Before proving Theorem 3.6, we show how it yields Theorem 10 of [15].

Corollary 3.7 ([15, Theorem 10]). Let $M$ be a semifinite von Neumann algebra on a separable Hilbert space, and let $\mathcal{K}$ be an ultraweakly dense 2-sided ideal in $M$. If $\mathcal{N}$ is a nest in $M$, then $\operatorname{alg}(\mathcal{N}) \cap \mathcal{K}$ is ultraweakly dense in $\operatorname{alg}(\mathcal{N}) \cap M$.

Proof. By Theorem 4.2.3 of [17] there is a uniformly continuous representation of $\mathbf{R}$, $\alpha=\left\{\alpha_{t}\right\}_{t \in \mathbf{R}}$, in the automorphism group of $M$ such that $\operatorname{alg}(\mathcal{N}) \cap M=M^{\alpha}[0, \infty)$. In fact, $\alpha$ is inner. Thus condition (ii) of Theorem 3.6 is satisfied. Because $\alpha$ is inner, $\alpha$ preserves every semifinite normal trace on $M$ and since $M$ acts on a separable Hilbert space, there is a faithful normal semifinite trace on $M$. Thus the condition (i) of Theorem 3.6 is satisfied. Theorem 3.6, then, completes the proof.

Proof of Theorem 3.6. We let $B=\mathcal{K}$ and $\pi$ be the identity representation in Corollary 3.5. We let $\alpha \mid \mathcal{K}$ be the $\alpha$ in that corollary and the $\beta$ of that corollary is our current $\alpha$. Let $e_{0}$ be the $\alpha$-finite projection for the system $(M, \alpha)$ and let $c$ be the central cover of $e_{0}$ in the center of $M$. By Corollary 3.5, we need only show that $e_{0} M^{\alpha}(0)$ is contained in the ultraweak closure of $c \cdot\left(M^{\alpha}(0) \cap \mathcal{K}\right)$. In fact, we show a little more. Let $q_{0}$ be the supremum of the finite projections in $M^{\alpha}(0) \cap \mathcal{K}$. (Recall that a projection $p$ is finite if and only if $\tau(p)<\infty$.) Of course $q_{0}$ may be zero. In any case, we show that $e_{0}=q_{0}$. This will complete the proof. But if $q$ is a finite projection in $M^{\alpha}(0)$ (if $q$ is finite, it must be in $\mathcal{K}$ ), and if $\varphi_{q}$ is defined by the formula $\varphi_{q}(a)=\tau(q a) / \tau(q)$, then a moment's reflection reveals that $\varphi_{q}$ is an invariant normal state on $M$ with $s\left(\varphi_{q}\right)=q$. By Lemma 3.1 (Part B), each such $q$ is dominated by $e_{0}$. Thus $q_{0} \leq e_{0}$. For the reverse inequality, define $\varphi$ on $M^{+}$by $\varphi(x)=\tau\left(x\left(e_{0}-q_{0}\right)\right), x \in M^{+}$. Then $\varphi$ is a normal, semifinite, invariant weight on $M^{+}$. (This follows since both $e_{0}$ and $q_{0}$ are in the center of $M^{\alpha}(0)$.) Evidently, $\varphi$ is supported by $e_{0}$ and so by Théorème 3.3 [7], the restriction of $\varphi$ to $M^{\alpha}(0)^{+}$is semifinite. Thus there is a $y \in M^{\alpha}(0)^{+}, y \neq 0$, such that $\varphi(y)<\infty$. This implies that $y\left(e_{0}-q_{0}\right)$ lies in $\mathcal{K} \cap M^{\alpha}(0)$. Hence for each $\epsilon>0$ the spectral projection, corresponding to $[\epsilon, \infty)$, of $y\left(e_{0}-q_{0}\right)$ lies in $\mathcal{K} \cap M^{\alpha}(0)$. Since these are all orthogonal to $q_{0}$ (recall $e_{0}$ and $q_{0}$ are in the center of $M^{\alpha}(0)$ and so the spectral projections of $y\left(e_{0}-q_{0}\right)$ are orthogonal to $\left.q_{0}\right)$, we contradict the fact that $q_{0}$ is the supremum of all finite projections in $M^{\alpha}(0)$, unless $y\left(e_{0}-q_{0}\right)=0$. Thus $y\left(e_{0}-q_{0}\right)=0$ for every $y \in M^{\alpha}(0)^{+}$such that $\varphi(y)<\infty$ and since these are ultraweakly dense in $M^{\alpha}(0)^{+}$, we conclude that $e_{0}=q_{0}$. 


\section{Coordinatized operator AlgEbras}

In this section we complement the results of [21] and show how the analysis in [14] and [1] relates to our perspective. For this purpose, we follow the notation of [21] and [19]. We fix a second countable, locally compact, Hausdorff, $r$-discrete, principal groupoid $G$. We view $G$ as an equivalence on $G^{(0)} \times G^{(0)}$. We write $\lambda=\left\{\lambda^{u}\right\}_{u \in G^{(0)}}$ for the Haar system on $G$, where $\lambda^{u}$ is counting measure on $G^{u}$, $u \in G^{(0)}$. (The assumption that such a Haar system exists is equivalent to assuming that the range and source maps, $r$ and $s$, are local homeomorphisms [24].) We assume, too, that $E$ is a $\mathbf{T}$-groupoid over $G$ and we write $j: E \rightarrow G$ for the quotient map. When convenient, we write $\dot{\alpha}$ for $j(\alpha)$. The space of compactly supported, continuous, complex-valued functions $f$ on $E$ such that $f(t \gamma)=t f(\gamma)$, $\gamma \in E, t \in \mathbf{T}$, becomes a $*$-algebra, denoted $C_{c}(G, E)$, under the operations

$$
(f * g)(\beta)=\int f(\alpha) g\left(\alpha^{-1} \beta\right) d \lambda^{r(\beta)}(\dot{\alpha})
$$

and

$$
f^{*}(\beta)=\overline{f\left(\beta^{-1}\right)} .
$$

The enveloping $C^{*}$-algebra of $C_{c}(G, E)$ will be denoted $C^{*}(G, E)$. This is the $C^{*}$-algebra $B$ that we fixed at the outset of this paper. We shall assume that $C^{*}(G, E)$ is nuclear, which is the same as assuming $G$ is measurewise amenable (see [19] and [24]). We fix once and for all a faithful cocycle $c: G \rightarrow \mathbf{R}$. This is a continuous homomorphism $c$ such that $G^{(0)}=c^{-1}(0)$. The cocycle $c$ determines a one-parameter group $\left\{\alpha_{t}\right\}_{t \in \mathbf{R}}$ on $B=C^{*}(G, E)$ by the formula

$$
\alpha_{t}(f)(\gamma)=e^{i t c(\dot{\gamma})} f(\gamma),
$$

$f \in C^{*}(G, E)$. (Note that our assumptions guarantee that the elements of $C^{*}(G, E)$ may be represented by continuous functions on $E$ that vanish at infinity. So the formula makes sense.) As is shown in [21], the algebra $A$ of analytic elements in $B=C^{*}(G, E)$ determined by $\left\{\alpha_{t}\right\}_{t \in \mathbf{R}}$ is $A(P(c))$, where $P(c):=\{\gamma \in E \mid c(\dot{\gamma}) \geq 0\}$ and $A(P(c)):=\left\{f \in C^{*}(G, E) \mid \operatorname{supp} f \subseteq P(c)\right\}$. We shall write $D$ for the diagonal of $A=A(P(c)), D=A \cap A^{*}$. Observe that since $c$ is assumed to be faithful $D=\left\{f \in C^{*}(G, E) \mid \operatorname{supp}(f) \subseteq j^{-1}\left(G^{(0)}\right)\right\}$ and this is canonically isomorphic to $C_{0}\left(G^{(0)}\right)$ because $E$, viewed as a principal T-bundle over $G$, is trivial over $G^{(0)}$. (See [16].)

A special class of examples deserves to be mentioned here. Let $\mathbf{Z}$ act freely on a locally compact Hausdorff space $X$ and write $x+n$ for the translate of $x$ by $n$. Let $G=X \times \mathbf{Z}$ with the usual groupoid structure: $(x, n)$ and $(y, m)$ are composable if and only if $y=x+n$ and $(x, n)(x+n, m):=(x, n+m)$, while $(x, n)^{-1}=(x+n,-n)$. The assumption that the $\mathbf{Z}$ action is free means that $G$ is isomorphic to the orbit equivalence relation in $X \times X:\{(x, x+n) \mid n \in \mathbf{Z}, x \in X\}$. The $\mathbf{T}$-groupoid in this example is trivial, i.e., $E=\mathbf{T} \times G$, and so we may ignore it in the formulas to follow. The $C^{*}$-algebra of $G$ in this case is usually denoted $C^{*}(X, \mathbf{Z})$ and is called the transformation group $C^{*}$-algebra determined by the action of $\mathbf{Z}$ on $X$. It is also called the crossed product determined by $\mathbf{Z}$ and $C_{0}(X)$. The cocycle $c$ in this example is the so-called position cocycle, $c(x, n)=n$. The set $P(c)$, then, may be viewed either as $X \times \mathbf{Z}_{+}$or as $\left\{(x, x+n) \mid n \in \mathbf{Z}_{+}, x \in X\right\}$, and the algebra $A$ is what is known in the literature as an analytic (or non-self-adjoint) crossed product.

Special cases of the following result appear in the literature $[5,17,20,18]$. 
Theorem 4.1. The algebra $A=A(P(c))$ is a strongly Dirichlet subalgebra of $C^{*}(G, E)$.

Proof. We need to show that $B^{\alpha}((-\infty, 0))+B^{\alpha}(0)+B^{\alpha}((0, \infty))$ is dense in $B=$ $C^{*}(G, E)$. First, recall that $B^{\alpha}(0)=\left\{f \in C^{*}(G, E) \mid \operatorname{supp}(f) \subseteq j^{-1}\left(G^{(0)}\right)\right\}$ because $c$ is faithful, and that $j^{-1}\left(G^{(0)}\right)$ is closed and open in $E$. Since $P(c) \cup$ $P(c)^{-1}=E$ and $P(c) \cap P(c)^{-1}=j^{-1}\left(G^{(0)}\right)$, we may write $E=j^{-1} c^{-1}(-\infty, 0) \cup$ $j^{-1} c^{-1}(0) \cup j^{-1} c^{-1}(0, \infty)$ where the sets in the union are disjoint and are closed and open. Of course these sets are also T-invariant. Furthermore, the analysis in [21] shows that $B^{\alpha}((0, \infty))=\left\{f \mid \operatorname{supp}(f) \subseteq j^{-1} c^{-1}(0, \infty)\right\}$ while $B^{\alpha}((-\infty, 0))=$ $\left\{f \mid \operatorname{supp}(f) \subseteq j^{-1} c^{-1}(-\infty, 0)\right\}$. Because each of the sets $j^{-1} c^{-1}(0, \infty), j^{-1} c^{-1}(0)$, and $j^{-1} c^{-1}(-\infty, 0)$ is closed and open, each $f \in C_{c}(G, E)$ may be written as $f=f_{-\infty}+f_{0}+f_{+\infty}$ where $f_{+\infty}$ lies in $C_{c}\left(j^{-1} c^{-1}(0, \infty)\right), f_{0} \in C_{c}\left(j^{-1} c^{-1}(0)\right)$, and $f_{-\infty} \in C_{c}\left(j^{-1} c^{-1}(-\infty, 0)\right)$. (Simply multiply $f$ by the characteristic functions of the various sets.) Since the sets are $\mathbf{T}$-invariant each of $f_{-\infty}, f_{0}$, and $f_{+\infty}$ transforms like $f$, i.e., each lies in $C_{c}(G, E)$. Since $C_{c}(G, E)$ is dense in $C^{*}(G, E)$, we conclude that $B^{\alpha}((-\infty, 0))+B^{\alpha}(0)+B^{\alpha}((0, \infty))$ is dense also.

A $C^{*}$-representation $\pi$ of $C^{*}(G, E)$ may be disintegrated and so each such $\pi$ is determined by a triple $\left(\mu, G^{(0)} * \mathcal{H}, U\right)$, where $\mu$ is a quasi-invariant measure on $G^{(0)}, G^{(0)} * \mathcal{H}$ is a Hilbert bundle on $G^{(0)}$, and $U: E \rightarrow \operatorname{Iso}\left(G^{(0)} * \mathcal{H}\right)$ is a Borel representation of $G$ in the isomorphism groupoid of the bundle, Iso $\left(G^{(0)} * \mathcal{H}\right)$ such that $U(t \gamma)=\bar{t} U(\gamma), t \in \mathbf{T}, \gamma \in E$. (See [24] and [25].) The exact relation between $\pi$ and $\left(\mu, G^{(0)} * \mathcal{H}, U\right)$ is expressed through the equation

$$
\pi(f) \xi(u)=\int f(\alpha)(U(\alpha) \xi)(u) \Delta^{1 / 2}(\alpha) d \lambda^{u}(\dot{\alpha}),
$$

where $f \in C_{c}(G, E), \xi$ is a square integrable section of the bundle, and where $\Delta$ is the modular function associated with $\mu$. (See [24].)

If the representation $\pi$ is irreducible or, more generally, if $\pi$ is factorial, then the measure $\mu$ is ergodic. In this event, we may form the asymptotic ranges of the cocycle $c$ calculated with respect to $\mu, R_{\infty}^{\mu}(c)$ and $\tilde{R}_{\infty}^{\mu}(c)$, as follows (see [26, Definition 3.1] and [21, Definition 2.3]):

1)

$$
\begin{aligned}
R_{\infty}^{\mu}(c)=\left\{t \in \mathbf{R} \mid \forall \epsilon>0 \text { and all } B \subseteq G^{(0)}\right. & , \mu(B)>0, \\
& \left.\left((B \times B) \cap c^{-1}(t-\epsilon, t+\epsilon)\right)>0\right\},
\end{aligned}
$$

where $\nu=\int \lambda^{u} d \mu(u)$ is the measure on $G$ induced by $\mu$ and $\left\{\lambda^{u}\right\}_{u \in G^{(0)}}$.

2) $\infty$ is an asymptotic value of $c$ if and only if for every $M>0$ and every $B \subseteq G^{(0)}, \mu(B)>0$, the inequality $\nu\left((B \times B) \cap c^{-1}[M, \infty)\right)>0$ holds.

3) We write $\tilde{R}_{\infty}^{\mu}(c)=R_{\infty}^{\mu}(c)$ if $\infty$ is not an asymptotic value of $c$ and we write $\tilde{R}_{\infty}^{\mu}(c)=R_{\infty}^{\mu}(c) \cup\{\infty\}$, if it is.

The following theorem is a blend of Theorem 1.2 and salient results of [21]. It refines [21, Theorems 3.2, 4.1, and 9.1].

Theorem 4.2. Let $\pi: C^{*}(G, E) \rightarrow \mathcal{L}\left(\mathcal{H}_{\pi}\right)$ be an irreducible representation and assume the associated measure is properly ergodic (i.e., not concentrated on an orbit). Let $\mathcal{A}$ be the ultraweak closure of $\pi(A)$. Then

1) either $\mathcal{A}=\mathcal{L}\left(\mathcal{H}_{\pi}\right)$ or $\mathcal{A}=\operatorname{alg}(\mathcal{N})$ for a continuous nest $\mathcal{N}$ in $\mathcal{L}\left(\mathcal{H}_{\pi}\right)$. 
2) If $\tilde{R}_{\infty}^{\mu}(c)=\{0, \infty\}$, then $\mathcal{A}=\mathcal{L}\left(\mathcal{H}_{\pi}\right)$.

3) If $c$ is $\mathbf{Z}$-valued, then $\mathcal{A}=\mathcal{L}\left(\mathcal{H}_{\pi}\right)$.

Proof. Theorem 3.2 of [21] implies that lat $\pi(A)$ is either $\{0, I\}$ or a continuous nest. Thus the first assertion follows from Theorem 1.2 and Theorem 4.1. Theorem 4.1 of [21] says that if $\tilde{R}_{\infty}^{\mu}(c)=\{0, \infty\}$, then lat $\pi(A)=\{0, I\}$. Therefore the second assertion follows from the first. Theorem 9.1 of [21] asserts that if $c$ is $\mathbf{Z}$-valued, then $\tilde{R}_{\infty}^{\mu}(c)=\{0, \infty\}$. Thus the third assertion follows from the first two.

Corollary 4.3. If $\pi: C^{*}(X, \mathbf{Z}) \rightarrow \mathcal{L}\left(\mathcal{H}_{\pi}\right)$ is an irreducible $C^{*}$-representation of $C^{*}(X, \mathbf{Z})$ and if the associated measure $\mu$ is properly ergodic, then $\pi(A)$ is ultraweakly dense in $\mathcal{L}\left(\mathcal{H}_{\pi}\right)$.

Recall that an algebra of operators $\mathcal{T}$ on a Hilbert space $\mathcal{H}$ is called triangular in case $\mathcal{T} \cap \mathcal{T}^{*}$ is a masa in $\mathcal{L}(\mathcal{H})$. This masa is called the diagonal of $\mathcal{T}$. Such an algebra is called maximal triangular iff it is not contained in any larger triangular algebra with the same diagonal and it is called irreducible iff it has no nontrivial invariant subspaces. In Theorem 2.2.1 of [14], Kadison and Singer showed that if $\mathcal{D}$ is an infinite dimensional masa on a Hilbert space $\mathcal{H}$ and if $U$ is a unitary operator on $\mathcal{H}$ that normalizes $\mathcal{D}$ and acts ergodically on $\mathcal{D}$, meaning that $U$ and $\mathcal{D}$ have no common, nontrivial, invariant subspaces, then the algebra $\mathcal{S}$ generated by $U$ and $\mathcal{D}$ is an irreducible triangular algebra with diagonal $\mathcal{D}$. So, therefore, every maximal triangular algebra $\mathcal{T}$ with diagonal $\mathcal{D}$ that contains $\mathcal{S}$ is also irreducible. In Theorem 3.3 of [2], Arveson showed that any algebra $\mathcal{A}$ on a (separable) Hilbert space $\mathcal{H}$ is weakly dense in $\mathcal{L}(\mathcal{H})$ if it has no invariant subspaces and contains a masa. Thus Kadison and Singer's algebras are weakly dense. Subsequently, in the remark on page 480 of [4] Arveson strengthened Theorem 3.3 of [2] by replacing "weakly dense" with "ultra weakly dense". Thus Kadison and Singer's algebras are ultraweakly dense in $\mathcal{L}(\mathcal{H})$.

This fact may also be derived from Corollary 4.3 as follows. Given $\mathcal{D}$ and $U$, it is well known that it is easy to produce a separable locally compact Hausdorff $X$, a homeomorphism $\tau$ of $X$, a quasi-invariant measure $\mu$ on $X$, and a unimodular function $\theta$ such that $\mathcal{D}$ is unitarily equivalent to the multiplication algebra determined by $L^{\infty}(X, \mu)$ acting on $L^{2}(X, \mu)$ through multiplication and $U$ is unitarily equivalent to the operator $\tilde{U}$ defined on $L^{2}(X, \mu)$ by the formula $(\tilde{U} \xi)(x)=\theta(x) \xi\left(\tau^{-1} x\right) J^{1 / 2}(x)$, where $J$ is the Radon-Nikodym derivative $d \mu \circ \tau / d \mu$. These ingredients are not uniquely determined by $\mathcal{D}$ and $U$, but that does not matter here. The action of $\mathbf{Z}$ on $X$ that $\tau$ produces need not be free, but if it isn't, $\mu$ assigns measure zero to the periodic points, and that is all that matters. Of course $\mu$ and $\theta$ determine an irreducible representation $\pi$ of $C^{*}(X, \mathbf{Z})$ on $L^{2}(X, \mu)$ by the formula

$$
(\pi(f) \xi)(x)=\sum_{k} f(x, k)\left(\tilde{U}^{k} \xi\right)(x)
$$

where $f \in C_{c}(X, \mathbf{Z})$ and $\xi \in L^{2}(X, \mu)$. Since the ultraweakly closed algebra generated by $\pi(A)$ contains the ultraweakly closed algebra generated by $\mathcal{D}$ (realized as $\left.L^{\infty}(X, \mu)\right)$ and $U($ realized as $\tilde{U})$, the latter is all of $\mathcal{L}(\mathcal{H})$ by Corollary 4.3.

In one sense, Arveson's approach to proving that ultraweak denseness of Kadison and Singer's algebras is preferable to ours. His analysis covers a much broader range of examples than those studied by Kadison and Singer and others. Nevertheless, it is noteworthy that Corollary 4.3 also produces operator algebras, whose diagonals 
have infinite multiplicity, that are ultraweakly dense in the full algebra of operators on the underlying Hilbert space. The ultraweak denseness of these algebras seems to be outside the scope of the techniques that Arveson developed.

We turn now to refinements of [21] that result when the irreducibility assumption on the representations studied is replaced by the assumption that they are factorial. As is customary, we shall identify subspaces with their orthogonal projections. We are interested in what one can say about factorial representations $\pi$ of $C^{*}(G, E)$ for which there is a nontrivial projection $Q$ in $M \cap$ lat $\pi(A)$ where, for the remainder of the paper, $M$ denotes $\pi\left(C^{*}(G, E)\right)^{\prime \prime}$.

Theorem 4.4. Let $\pi: C^{*}(G, E) \rightarrow \mathcal{L}(\mathcal{H})$ be a $C^{*}$-representation and assume $M=$ $\pi\left(C^{*}(G, E)\right)^{\prime \prime}$ is a factor. Further, suppose there is a projection in $M \cap \operatorname{lat}(\pi(A))$ that is different from 0 and $I$. Then:

1) $\pi$ is protocovariant, and there is a unitary representation $U=\left\{U_{t}\right\}_{t \in \mathbf{R}}$ in $M$, such that $(\pi, U)$ is a covariant representation of $(B, \mathbf{R}, \alpha)^{2}$.

2) If $V=\left\{V_{t}\right\}_{t \in \mathbf{R}}$ is a unitary representation of $\mathbf{R}$ on $\mathcal{H}$ such that $(\pi, V)$ is covariant and if $\tilde{\alpha}_{t}$ is $A d V_{t}$ acting on $M$, then the ultraweak closure of $\pi(A)$ is $M^{\tilde{\alpha}}([0, \infty))$.

3) If $U=\left\{U_{t}\right\}_{t \in \mathbf{R}}$ is a unitary representation of $\mathbf{R}$ in $M$ such that $(\pi, U)$ is covariant, and if $U_{t}=\int e^{i \lambda t} d E(\lambda)$ is the spectral representation of $U$, then lat $\pi(A) \cap M$ is the set of projections $\mathcal{N}=\{0, E(\lambda, \infty), E[\lambda, \infty), I\}_{\lambda \in \mathbf{R}}$.

4) The ultraweak closure of $\pi(A)$ is a nest subalgebra of $M$, namely $M \cap \operatorname{alg}(\mathcal{N})$, where $\mathcal{N}$ is as in 3 ).

$5)$ In the notation of 3), each atom of $E$ is a minimal projection in $M$. Thus, if $M$ is a continuous factor, $E$ is nonatomic and $\mathcal{N}$ is continuous nest.

Proof. Fix $Q \in M \cap \operatorname{lat}(\pi(A))$ that is different from 0 and $I$. Form the projections $Q_{t}$ onto the subspaces $\left[B^{\alpha}[t, \infty) Q \mathcal{H}\right], t \in \mathbf{R}$, where the brackets denote closure of what is inside and where, recall, $B=C^{*}(G, E)$. Evidently, by the double commutant theorem, each $Q_{t}$ lies in $M$ and by [17, Corollary 2.15], $Q_{t} \in$ lat $\pi(A)$. If $Q_{-\infty}=\bigvee_{t \in \mathbf{R}} Q_{t}$ and $Q_{\infty}=\bigwedge_{t \in \mathbf{R}} Q_{t}$, then $Q_{ \pm \infty}$ reduces $M$ (see the proof of [17, Theorem 5.2]). Since $M$ is assumed to be a factor and $Q$ is neither 0 nor $I$, we conclude that $Q_{-\infty}=I$ and $Q_{+\infty}=0$. As in the proof of Theorem 5.2 of [17], we conclude that there is a spectral measure $E$ in $M$ such that for all $\lambda \in \mathbf{R}$, $E[\lambda, \infty)=\bigwedge_{t<\lambda} Q_{t}$. If $U_{t}=\int e^{i \lambda t} d E(\lambda)$ then, as is shown in [17, Theorem 5.2], $(\pi, U)$ is a covariant representation. Of course $U$ lies in $M$ because its spectral projections are in $M$. Thus part 1) is proved.

Of course part 2) is an immediate consequence of Theorem 2.9 and part 1). It is worthwhile to note that the ultraweak closure of $\pi(A)$ is independent of any ultraweakly continuous representation of $\mathbf{R}$ on $M,\left\{\beta_{t}\right\}_{t \in \mathbf{R}}$, such that $\beta_{t} \circ \pi=\pi \circ \alpha_{t}$.

Parts 3), 4) and 5) all rest on showing that the atoms of $E$ are minimal projections in $M$. This, in turn, uses an argument found in the proof of Theorem 2.9. So let $\lambda$ be an atom of $E$. We show $E(\{\lambda\}) M E(\{\lambda\})$ is contained in the ultraweak closure of $E(\{\lambda\}) \pi(D) E(\{\lambda\})$. Note that $\pi(D)$ commutes with $E$ because $\alpha$ fixes $D$ elementwise and $A d U_{t} \circ \pi=\pi \circ \alpha_{t}$. This shows that $\pi(D)$ commutes with $U$ and, therefore, with $E$. Since $\pi(D)$ is abelian, this will show that $E(\{\lambda\})$ is an abelian projection. Since $M$ is a factor we will conclude that $E(\{\lambda\})$ is a minimal projection. By [17, Corollary 2.14], we see that $E(\{\lambda\}) \pi(b) E(\{\lambda\})=0$ if $b$ lies

\footnotetext{
${ }^{2}$ In this event, we say $\alpha$ is inner.
} 
either in $B^{\alpha}(0, \infty)$ or in $B^{\alpha}(-\infty, 0)$. Since $D=B^{\alpha}(0)$, the assertion is immediate from Theorem 4.1. Part 5) now has been proved. Part 4) follows from part 3), part 2) and [17, Corollary 2.14]. So it remains to prove part 3). However, if $P$ is a projection in $M \cap \operatorname{lat}(\pi(A))$, then the arguments just presented yield a unitary representation $\left\{V_{t}\right\}_{t \in \mathbf{R}}$ in $M$ with associated spectral measure $F$ in $M$ such that $F([0, \infty)) \geq P \geq F((0, \infty))$. The projection $F(\{0\})$ is also a minimal projection in $M$, so $P$ either equals $F([0, \infty))$ or $F((0, \infty))$. But, since $(\pi, U)$ and $(\pi, V)$ are covariant representations, we conclude that for each $t \in \mathbf{R}, U_{t} V_{t}^{*}$ commutes with $M$. Since these operators lie in $M$, we find that there is a scalar $\lambda(t)$ such that $V_{t}=\lambda(t) U_{t}$. A moment's reflection reveals that $\lambda$ is, in fact, a continuous character and so there is an $r \in \mathbf{R}$ such that $\lambda(t)=e^{i t r}$. This, in turn, implies that $F[t, \infty)=E[t-r, \infty)$ for all $t$ and shows that $P$ is either $E([-r, \infty))$ or $E((-r, \infty))$.

Let $\pi$ be a factorial representation of $C^{*}(G, E)$ on a Hilbert space $\mathcal{H}_{\pi}$, let $\left(\mu, G^{(0)} * \mathcal{H}, U\right)$ be the data associated to the disintegration of $\pi$ and let $N$ be a nontrivial subspace in $M \cap \operatorname{lat}(\pi(A))$. Then $N$ reduces $\pi(D)$ and so, when viewed as a projection, is decomposable. The invariance of $N$ translates into the equation

$$
U(\gamma) N(s(\gamma)) U(\gamma)^{*} \leq N(r(\gamma))
$$

for almost all $\gamma \in E$. (The measure on $E$ is the natural measure induced by Haar measure on $\mathbf{T}$, the Haar system $\lambda$, and $\mu$. See [21, Lemma 3.1].) The fact that $\pi$ is factorial means that $\mu$ is ergodic. The proof of the following result, then, follows the same line of argument spelled out in [21], using Theorem 4.4, here, to replace Theorem 3.2 of [21]. We shall therefore only indicate briefly what changes are necessary in the arguments of [21] to complete the proof.

Theorem 4.5. Let $\pi$ be a factorial representation of $C^{*}(G, E)$ and assume there is a nontrivial $N$ in $\mathcal{N}=M \cap \operatorname{lat}(\pi(A))$, where $M=\pi\left(C^{*}(A, E)\right)^{\prime \prime}$. If $\mu$ is the quasi-invariant measure associated to $\pi$, then:

1) $\tilde{R}_{\infty}^{\mu}(c) \neq\{0, \infty\}$.

2) If $\tilde{R}_{\infty}^{\mu}(c)=\{0\}$, so that $c$ is a Borel coboundary with respect to the measure $\mu^{3}$, then $\mathcal{N}$ is contained in $\pi(D)^{\prime \prime}$.

3) If $\tilde{R}_{\infty}^{\mu}(c)=\lambda \mathbf{Z}$, then for $\mu$-almost every $e \in G^{(0)},\{N(u) \mid N \in \mathcal{N}\}$ is order isomorphic to $\mathbf{Z} \cup\{ \pm \infty\}$.

4) If $\tilde{R}_{\infty}(c)=\mathbf{R}$, then for $\mu$-almost every $u \in G^{(0)},\{N(u) \mid N \in \mathcal{N}\}$ is order isomorphic to $\mathbf{R} \cup\{ \pm \infty\}$.

Proof. Part 1) is the analogue of Theorem 4.1 of [21], stated contrapositively, since $\mathcal{N}$ is assumed to be nontrivial. In the proof of [21, Theorem 4.1] we used only ergodicity and Proposition 3.3 of [21]. That proposition in turn used only [21, Theorem 3.2] which implies, when $\pi$ is irreducible and the measure $\mu$ is properly ergodic, that lat $\pi(A)$ is a continuous nest. However, it is easy to see that in the factorial case, one may replace lat $\pi(A)$ by $\mathcal{N}=M \cap$ lat $\pi(A)$ and then Theorem 4.4 completes the proof. Part 2) is the "factorial analogue" of Theorem 5.1 of [21]. All we need, when $\mu$ is properly ergodic, is the fact that $\mathcal{N}$ is a continuous nest. This follows from Theorem 4.4. Part 3) is an analogue of Corollary 6.6 of [21] and rests on a modified form of [21, Theorem 6.1]. One has to replace lat $\pi(\mathcal{T}(P))$ there

\footnotetext{
${ }^{3}$ This means that there is a Borel function $b$ on $G^{(0)}$ such that on $G, c(\dot{\gamma})=b(r(\gamma)) b(s(\gamma))^{-1}$ a.e. $\nu$.
} 
by lat $\pi(\mathcal{T}(P)) \cap M$ where $\mathcal{T}(P)$ is defined like $A$, but in the Borel category where the cocycle $c$ defining $P$ is assumed only to be Borel. In the last line of the proof of [21, Theorem 6.1], where we used irreducibility, it is enough to assume that $\pi$ is factorial and that the projections $F_{t}$, produced there, lie in $M$. Corollary 6.6 of [21] also requires the discussion after Lemma 6.2 and Corollary 6.3 of [21]. The fact that there are $t_{n}$ 's such that $F_{n}=Q_{t_{n}}$ (notation of [21]) in the factorial case follows from Theorem 4.4. It also follows that $L_{n} \in M$ and that $L_{n}$ is a minimal projection in $M \cap \pi\left(M_{n}\right)^{\prime}$ just as was proved there. It results that $\pi_{n}$ is factorial and this replaces Corollary 6.3 in [21]. Finally, part 4, really, is an analogue of [21, Theorem 7.1] and the modifications of the proof are not substantial.

One can define $\tilde{R}_{\infty}(c)$ (see [24] or the discussion before Definition 2.3 in [21]) and conclude just as in the proof of Theorem 9.1 of [21] that $\tilde{R}_{\infty}^{\mu}(c) \subseteq \tilde{R}_{\infty}(c)$ for each quasi-invariant ergodic measure $\mu$ and that if $c$ is $\mathbf{Z}$-valued, then either $\tilde{R}_{\infty}^{\mu}(c)=\{0, \infty\}$ or $\tilde{R}_{\infty}^{\mu}(c)=\{0\}$. In the second case, of course, $\mu$ is concentrated on an orbit, since $\mu$ is assumed to be ergodic. The arguments for [21, Theorem 9.1] imply, mutatis mutandis, using Theorem 4.5, the following corollary.

Corollary 4.6. (1) If $\tilde{R}_{\infty}(c) \subseteq\{0, \infty\}$ and if $\pi$ is a factorial representation of $C^{*}(G, E)$ for which $\mathcal{N}:=M \cap$ lat $\pi(A)$ is not trivial, then $\mathcal{N} \subseteq \pi(D)^{\prime \prime}$.

(2) If, furthermore, $c$ is $\mathbf{Z}$-valued, $\mu$ must be concentrated on an orbit. In this event, $\mathcal{N}$ is a totally atomic nest, ordered as the orbit that carries $\mu$.

One can weaken the factorial assumption on $\pi$ in Corollary 4.6 and assume that there is a nontrivial projection $Q$ in $M \cap$ lat $\pi(A)$ such that the central supports of $Q$ and $I-Q$ in $M$ are $I$. Such a $\pi$ is the direct integral of factorial representations of the kind considered in Corollary 4.6. We call such a representation an $N$-representation to indicate a connection with nests. The following corollary is an immediate consequence of Corollary 4.6.

Corollary 4.7. If $\tilde{R}_{\infty}(c) \subseteq\{0, \infty\}$, then for every $N$-representation $\pi, M \cap$ $\operatorname{lat}(\pi(A)) \subseteq \mathcal{Z}(M) \vee \pi(D)^{\prime \prime}$, where $\mathcal{Z}(M)$ is the center of $M$. If, moreover, $c$ is $\mathbf{Z}$-valued then every $N$-representation is type $I$.

One further corollary of Theorem 4.5 should be cited.

Corollary 4.8. Suppose that $\pi$ is a factorial representation of $C^{*}(G, E)$ on a Hilbert space $\mathcal{H}$ and suppose that $M:=\pi\left(C^{*}(G, E)\right)^{\prime \prime}$ is a finite factor. If $\mathcal{N}=$ $M \cap$ lat $\pi(A)$ is nontrivial, then the cocycle $c$ is a Borel coboundary with respect to the measure $\mu$.

Proof. By Theorem 4.4 there is a unitary representation $\left\{U_{t}\right\}_{t \in \mathbf{R}}$ in $M$ so that $\tilde{\alpha}_{t} \circ \pi=\pi \circ \alpha_{t}$, where $\tilde{\alpha}_{t}=A d U_{t}$, and such that $\mathcal{N}^{\prime \prime}=\left\{U_{t}\right\}_{t \in \mathbf{R}}^{\prime \prime}$. It follows that $\mathcal{N} \subseteq M^{\tilde{\alpha}}(0)$ and that there is an $\tilde{\alpha}$-invariant, faithful, normal, conditional expectation $\epsilon: M \rightarrow M^{\tilde{\alpha}}(0)$, i.e., $M$ is $\tilde{\alpha}$-finite. Hence by Theorem 3.4, $M^{\tilde{\alpha}}(0)$ is the ultraweak closure of $\pi(D)$ and so $\mathcal{N} \subseteq \pi(D)^{\prime \prime}$. By Theorem 4.5, $\tilde{R}_{\infty}^{\mu}(c)=\{0\}$ so that $c$ is a Borel coboundary.

\section{REFERENCES}

[1] Wm. B. Arveson, Operator algebras and measure preserving automorphisms, Acta Math. 118 (1967), 95 - 109. MR 35:1751

[2] Wm. B. Arveson, A density theorem for operator algebras, Duke Math. J. 34 (1967), 635 647. MR 36:4345 
[3] Wm. B. Arveson, On groups of automorphisms of operator algebras, J. Functional Analysis 15 (1974), 217 - 243. MR 50:1016

[4] Wm. B. Arveson, Operator algebras and invariant subspaces, Ann. Math. 100 (1974), 433 532. MR 51:1420

[5] Wm. B. Arveson and K. B. Josephson, Operator algebras and measure preserving automorphisms, II, J. Functional Analysis 4 (1969), 100 - 134. MR 40:3322

[6] O. Bratteli and D. W. Robinson, Operator Algebras and Quantum Statistical Mechanics I, Springer-Verlag, New York - Heidelberg - Berlin, 1979. MR 81a:46070

[7] Dang-Ngoc-Nghiem, Sur la classification des systèmes dynamiques non commutatifs, J. Functional Analysis 15 (1974), 188 - 201. MR 50:1007

[8] J. Dixmier, Les Algèbres d'Opérateurs dans l'espace Hilbertien, Gauthier - Villars, Paris, 1969. MR 50:5482

[9] J. Dixmier, Les $C^{*}$-algèbres et leurs représentations, Gauthier - Villars, Paris, 1964. MR 39:7442

[10] A. Guichardet, Systèmes dynamiques non comutatifs, Societé Mathématique de France Asterisque 13 - 14, 1974. MR 50:5483

[11] U. Haagerup, The standard form of a von Neumann algebra, Math. Scand. 37 (1975), 271 283. MR 53:11387

[12] H. Halpern, Unitary implementation of automorphism groups on von Neumann algebras, Comm. Math. Physics 25 (1972), 253 - 275. MR 45:5768

[13] P. E. T. Jørgensen, Ergodic properties of one-parameter automorphism groups of operator algebras, J. Math. Anal. Appl. 87 (1982), 354 -372. MR 83i:46079

[14] R. V. Kadison and I. M. Singer, Triangular operator algebras, Amer. J. Math. 82 (1960), 227 - 259. MR 22:12409

[15] V. Kaftal, D. R. Larson, and G. Weiss, Quasitriangular subalgebras of semifinite von Neumann algebras are closed, J. Functional Analysis 107 (1992), 387 - 401. MR 93g:46060

[16] A. Kumjian, On $C^{*}$-diagonals, Can. J. Math. 38 (1986), 969 - 1008. MR 88a:46060

[17] R. I. Loebl and P. S. Muhly, Analyticity and flows in von Neumann algebras, J. Functional Analysis 29 (1978), 214 -252. MR 81h:46080

[18] P. S. Muhly, Radicals, crossed products, and flows, Ann. Polon. Math. 43 (1983), 35 - 42. MR 85i:46093

[19] P. S. Muhly, C. Qiu, and B. Solel, Coordinates, nuclearity and spectral subspaces in operator algebras, J. Operator Theory 26 (1991), 313 - 332. MR 94i:46075

[20] P. S. Muhly, C. Qiu, and J. Xia, Analyticity, uniform averaging, and K-theory, in Algebraic Methods in Operator Theory, Raúl Curto and Palle E. T. Jørgensen, eds., Birkhäuser, Boston, 1994, 328 - 349. MR 95j:46083

[21] P. S. Muhly and B. Solel, Representations of triangular subalgebras of groupoid $C^{*}$-algebras, to appear in J. Australian Math. Soc.

[22] J. L. Orr and J. Peters, Some representations of TAF algebras, Pacific J. Math. 167 (1995), 129-161. MR 96c:46055

[23] G. Pedersen, $C^{*}$-algebras and their Automorphism Groups, Academic Press, London - New York - San Francisco, 1979. MR 81e:46037

[24] J. N. Renault, A Groupoid Approach to $C^{*}$-algebras, Springer LNM \#793, Berlin - Heidelberg - New York, 1980. MR 82h:46075

[25] J. N. Renault, Représentations des produits croisés d'algèbres de groupoïdes, J. Operator Theory 18 (1987), 67 - 97. MR 89g:46108

[26] K. Schmidt, Cocycles on ergodic transformation groups, Macmillan, Lectures in Mathematics, \#1, The Macmillan Company of India, 1977. MR 58:28262

[27] L. Zsidó, Spectral and ergodic properties of analytic generators, J. Approx. Theory 20 (1977), 77 - 138. MR 57:3912

Department of Mathematics, The University of Iowa, Iowa City, Iowa 52242

E-mail address: muhly@math.uiowa.edu

Department of Mathematics, Technion - Israel Institute of Technology, Haifa 32000 , ISRAEL

E-mail address: mabaruch@techunix.technion.ac.il 